\title{
The Mumford-Tate conjecture for products of abelian varieties
}

\author{
Johan Commelin
}

\begin{abstract}
Let $X$ be a smooth projective variety over a finitely generated field $K$ of characteristic 0 , and fix an embedding $K \subset \mathbb{C}$. The Mumford-Tate conjecture is a precise way of saying that a certain extra structure on the $\ell$-adic étale cohomology groups of $X$ (namely, a Galois representation) and a certain extra structure on the singular cohomology groups of $X$ (namely, a Hodge structure) convey the same information.

The main result of this paper says that if $A_{1}$ and $A_{2}$ are abelian varieties (or abelian motives) over $K$ and the Mumford-Tate conjecture holds for both $A_{1}$ and $A_{2}$, then it holds for $A_{1} \times A_{2}$. These results do not depend on the embedding $K \subset \mathbb{C}$.
\end{abstract}

\section{Introduction}

1.1. Let $A$ be an abelian variety over a finitely generated field $K \subset \mathbb{C}$. Denote by $\bar{K}$ the algebraic closure of $K$ in $\mathbb{C}$. If $\ell$ is a prime number, we write $\mathrm{H}_{\ell}^{1}(A)$ for the $\ell$-adic cohomology group $\mathrm{H}_{\text {ét }}^{1}\left(A_{\bar{K}}, \mathbb{Q}_{\ell}\right)$. Similarly, we write $\mathrm{H}_{\mathrm{B}}^{1}(A)$ for the singular cohomology group $\mathrm{H}_{\text {sing }}^{1}(A(\mathbb{C}), \mathbb{Q})$. There is a natural isomorphism $\mathrm{H}_{\ell}^{1}(A) \cong \mathrm{H}_{\mathrm{B}}^{1}(A) \otimes \mathbb{Q}_{\ell}$ of vector spaces.

The vector space $\mathrm{H}_{\ell}^{1}(A)$ carries a Galois representation $\rho_{\ell}: \operatorname{Gal}(\bar{K} / K) \rightarrow \operatorname{GL}\left(\mathrm{H}_{\ell}^{1}(A)\right)$, while $\mathrm{H}_{\mathrm{B}}^{1}(A)$ carries a Hodge structure. This Hodge structure may be described by a representation $\rho: \mathrm{G}_{\mathrm{B}}(A) \rightarrow \mathrm{GL}\left(\mathrm{H}_{\mathrm{B}}^{1}(A)\right)$, where $\mathrm{G}_{\mathrm{B}}(A)$ is the Mumford-Tate group of $A$ (see Section 3 ).

Write $\mathrm{G}_{\ell}(A)$ for the Zariski closure of the image of $\rho_{\ell}$ and $\mathrm{G}_{\ell}^{\circ}(A)$ for the identity component of $\mathrm{G}_{\ell}(A)$. The Mumford-Tate conjecture expresses the expectation that the comparison isomorphism $\mathrm{H}_{\ell}^{1}(A) \cong \mathrm{H}_{\mathrm{B}}^{1}(A) \otimes \mathbb{Q}_{\ell}$ identifies $\mathrm{G}_{\ell}^{\circ}(A)$ with $\mathrm{G}_{\mathrm{B}}(A) \otimes \mathbb{Q}_{\ell}$. This conjecture is still wide open.

1.2. Main theorem. The goal of this article is to prove Theorem 10.5:

Let $A_{1}$ and $A_{2}$ be two abelian varieties over a finitely generated field $K \subset \mathbb{C}$. If the Mumford-Tate conjecture is true for $A_{1}$ and $A_{2}$, then it is true for $A_{1} \times A_{2}$.

In fact, in Theorem 10.3, we prove the more general statement that the full subcategory of abelian motives over $K$ consisting of motives for which the Mumford-Tate conjecture holds is a

Received 30 April 2018, accepted in final form 14 September 2018.

2010 Mathematics Subject Classification 14F20, 14K15.

Keywords: Mumford-Tate conjecture, motives, abelian varieties.

This journal is (C) Foundation Compositio Mathematica 2019. This article is distributed with Open Access under the terms of the Creative Commons Attribution Non-Commercial License, which permits non-commercial reuse, distribution, and reproduction in any medium, provided that the original work is properly cited. For commercial re-use, please contact the Foundation Compositio Mathematica.

This research has been financially supported by the Netherlands Organisation for Scientific Research (NWO) under projects no. 613.001.207 (Arithmetic and motivic aspects of the Kuga-Satake construction) and no. 613.001 .651 (The Cohomology of the Moduli Space of Curves) and by the Deutsche Forschungs Gemeinschaft (DFG) under Graduiertenkolleg 1821 (Cohomological Methods in Geometry). 


\section{MTC FOR PRODUCTS OF ABELIAN VARIETIES}

subcategory that is closed under direct sums, tensor products, duals, and direct summands.

Remark 1.3. (1) Observe that the conclusion of the theorem is not a formal consequence of the assumption: Suppose that $G^{\prime}$ is a group, with two representations $\rho_{1}: G^{\prime} \rightarrow \operatorname{GL}\left(V_{1}\right)$ and $\rho_{2}: G^{\prime} \rightarrow \operatorname{GL}\left(V_{2}\right)$. Let $G_{1}$ and $G_{2}$ be the images of $\rho_{1}$ and $\rho_{2}$, respectively. Write $\rho$ for $\rho_{1} \oplus \rho_{2}$, and let $G$ be the image of $\rho$. Then $G$ is a subgroup of $G_{1} \times G_{2}$, and the projection of $G$ onto $G_{1}$ (or $G_{2}$ ) is surjective. However, $G \subset G_{1} \times G_{2}$ may be anything, ranging from the diagonal (for example, if $V_{1} \cong V_{2}$ ) to the full product (for example, if $G_{1} \neq G_{2}$ and both groups are simple).

In the context of the main theorem, we have

$$
\mathrm{G}_{\ell}^{\circ}\left(A_{1} \times A_{2}\right) \subset \mathrm{G}_{\ell}^{\circ}\left(A_{1}\right) \times \mathrm{G}_{\ell}^{\circ}\left(A_{2}\right) \cong\left(\mathrm{G}_{\mathrm{B}}\left(A_{1}\right) \times \mathrm{G}_{\mathrm{B}}\left(A_{2}\right)\right) \otimes \mathbb{Q}_{\ell} \supset \mathrm{G}_{\mathrm{B}}\left(A_{1} \times A_{2}\right) \otimes \mathbb{Q}_{\ell},
$$

and there is no a priori formal reason why $\mathrm{G}_{\ell}^{\circ}\left(A_{1} \times A_{2}\right)$ and $\mathrm{G}_{\mathrm{B}}\left(A_{1} \times A_{2}\right) \otimes \mathbb{Q}_{\ell}$ should be the same subgroup.

(2) The situation above is exactly the setup where Goursat's lemma applies: we have two groups $G_{1}$ and $G_{2}$ and a subgroup $G^{\prime} \subset G_{1} \times G_{2}$ such that the projections $\pi_{i}: G \rightarrow G_{i}$ are surjective $(i=1,2)$. Let $N_{1}$ be the kernel of $\pi_{2}$ and $N_{2}$ the kernel of $\pi_{1}$. Goursat's lemma is the observation that one may identify $N_{i}$ with a normal subgroup of $G_{i}$, and the image of $G^{\prime}$ in $G_{1} / N_{1} \times G_{2} / N_{2}$ is the graph of an isomorphism $G_{1} / N_{1} \rightarrow G_{2} / N_{2}$.

This lemma is also true in the context of algebraic groups, a fact that we will need later on. We leave the proofs of these statements as an exercise to the reader.

Remark 1.4. This paper extends work of Lombardo [Lom16] and Vasiu [Vas08]. The latter reference contains a result similar to Theorem 10.5, although it has to exclude the case where $A_{1}$ or $A_{2}$ has a Mumford-Tate group with a simple factor of type $\mathrm{D}_{4}^{\mathbb{H}}$. Its proof is long and very technical, and I do not claim to grasp the details. Vasiu's global strategy is similar to the one employed below, and the reason that we can now prove the stronger claim is mostly due to the results of [Com17] (building on [Kis17]).

\subsection{Strategy of the proof}

(1) As a first step, we linearise the category of abelian varieties into so-called abelian motives (in the sense of André [And96b] or motives for absolute Hodge cycles). We obtain a semisimple Tannakian category, allowing us to apply the toolkit of the representation theory of reductive linear groups.

(2) From work of several people (notably Piatetski-Shapiro, Deligne, André, and Faltings) we know that for any abelian motive $M$, the group $\mathrm{G}_{\ell}^{\circ}(M)$ is reductive, and we have an inclusion $\mathrm{G}_{\ell}^{\circ}(M) \subset \mathrm{G}_{\mathrm{B}}(M) \otimes \mathbb{Q}_{\ell}$.

(3) We then prove that the connected component of the centre of $\mathrm{G}_{\ell}^{\circ}(A)$ is isomorphic to the connected component of the centre of $\mathrm{G}_{\mathrm{B}}(A) \otimes \mathbb{Q}_{\ell}$. For this we employ CM motives (see Section 4.16) and reduce the claim to the Mumford-Tate conjecture for CM abelian varieties, which is known by work of Pohlmann [Poh68]. (This result is proven in [Vas08, Theorem 1.3.1] and [UY13, Corollary 2.11] using different methods.)

(4) The next step consists of replacing the abelian variety $A_{i}(i=1,2)$ with the motive $M_{i}$ that corresponds - via the Tannakian formalism - to the adjoint representation of $\mathrm{G}_{\mathrm{B}}\left(A_{i}\right)^{\text {ad }}$. It suffices to prove the Mumford-Tate conjecture for $M_{1} \oplus M_{2}$.

(5) By general considerations, we may assume that $M_{1}$ and $M_{2}$ are irreducible motives. In particular, the Mumford-Tate groups $\mathrm{G}_{\mathrm{B}}\left(M_{1}\right)$ and $\mathrm{G}_{\mathrm{B}}\left(M_{2}\right)$ are $\mathbb{Q}$-simple adjoint groups. In addition, we assume $\mathrm{G}_{\ell}^{\circ}\left(M_{1} \oplus M_{2}\right) \mp \mathrm{G}_{\ell}^{\circ}\left(M_{1}\right) \times \mathrm{G}_{\ell}^{\circ}\left(M_{2}\right)$. 


\section{J. Commelin}

(6) We use Goursat's lemma (see Remark 1.3(ii)) and results from [Com17] to show that for all prime numbers $\ell$, we have $\mathrm{H}_{\ell}\left(M_{1}\right) \cong \mathrm{H}_{\ell}\left(M_{2}\right)$. From this, we deduce that there is a canonical isomorphism $\operatorname{End}\left(M_{1}\right) \cong \operatorname{End}\left(M_{2}\right)$.

(7) The remainder of the proof consists in applying to $M_{1}$ and $M_{2}$ a construction of Deligne that is reminiscent of the Kuga-Satake construction for K3 surfaces. As a result, we acquire two abelian varieties $\tilde{A}_{1}$ and $\tilde{A}_{2}$, and our job is to show that the isomorphism $\mathrm{H}_{\ell}\left(M_{1}\right) \cong \mathrm{H}_{\ell}\left(M_{2}\right)$ lifts to an isomorphism $\mathrm{H}_{\ell}^{1}\left(\tilde{A}_{1}\right) \cong \mathrm{H}_{\ell}^{1}\left(\tilde{A}_{2}\right)$.

(8) Once that is done, we apply Faltings's theorem to deduce that $\tilde{A}_{1}$ and $\tilde{A}_{2}$ are isogenous abelian varieties. This in turn implies $\mathrm{G}_{\mathrm{B}}\left(\tilde{A}_{1}\right) \cong \mathrm{G}_{\mathrm{B}}\left(\tilde{A}_{2}\right)$. In particular, $\mathrm{G}_{\mathrm{B}}\left(M_{1} \oplus M_{2}\right) \subset \mathrm{G}_{\mathrm{B}}\left(M_{1}\right) \times$ $\mathrm{G}_{\mathrm{B}}\left(M_{2}\right)$ is the diagonal; hence, $\mathrm{G}_{\ell}^{\circ}\left(M_{1} \oplus M_{2}\right) \cong \mathrm{G}_{\mathrm{B}}\left(M_{1} \oplus M_{2}\right) \otimes \mathbb{Q}_{\ell}$, and we are done.

Notation 1.6. For any field $K$, we denote the absolute Galois group $\operatorname{Gal}(\bar{K} / K)$ by $\Gamma_{K}$.

\section{Hyperadjoint objects in Tannakian categories}

In representation theory, the adjoint representation is very important. Via the Tannakian formalism, we port adjoint representations to Tannakian categories. (For a good overview of the Tannakian formalism, see [Bre94]. For details, we refer to [Del90] and [DMOS82].)

This leads to the definition of hyperadjoint objects in Tannakian categories. We study some of their properties in Lemma 2.11. This section ties into the proof of the main theorem because we will replace an abelian variety $A$ with the motive that corresponds to the adjoint representation of the motivic Galois group of $A$. (See also the strategy in Section 1.5.)

2.1. Let $Q$ be a field of characteristic 0 , and let $T$ be a $Q$-linear symmetric monoidal category. Let $R$ be a $Q$-algebra, and denote the category of finitely generated projective $R$-modules by $\operatorname{Proj}_{R}$. An $R$-valued fibre functor of $T$ is a $Q$-linear monoidal functor $T \rightarrow \operatorname{Proj}_{R}$ that is faithful and exact. We denote the groupoid of fibre functors $T \rightarrow \operatorname{Proj}_{R}$ by $\operatorname{Fib}(T)_{R}$.

2.2. Let $Q$ be a field of characteristic 0 . A Tannakian category over $Q$ is a $Q$-linear rigid abelian symmetric monoidal category with an isomorphism $Q \stackrel{\sim}{\rightarrow} \operatorname{End}(1)$ such that for every object $V \in T$, the following equivalent conditions hold: (1) there exists an integer $n$ such that $\wedge^{n} V=0$; or $(2) \operatorname{dim}(V)$ is an integer. (See [Del90, $\S 1.2$ and théorème 7.1].) The exterior power $\wedge^{n} V$ is defined in the usual way in terms of $\otimes^{n} V$ and antisymmetrisation. The dimension of $V$ is defined as the trace of the identity morphism on $V$; in other words, $\operatorname{dim}(V)$ is the composition

of the natural morphisms $\delta$ (unit) and ev (counit): $1 \stackrel{\delta}{\longrightarrow} V^{\star} \otimes V \stackrel{\text { ev }}{\longrightarrow} 1$. Théorème 7.1 of [Del90] shows that the two conditions listed above are equivalent to the existence of a $Q$-algebra $R$ and a fibre functor $T \rightarrow \operatorname{Proj}_{R}$, respectively.

2.3. Throughout the rest of this section, $T$ will denote a Tannakian category over a field $Q$ of characteristic 0. For a $Q$-algebra $R$, recall that $\operatorname{Fib}(T)_{R}$ is the groupoid of fibre functors $T \rightarrow \operatorname{Proj}_{R}$. It turns out that $\mathrm{Fib}(T)$ is an algebraic stack over $Q$. In fact, if $\alpha: Q \rightarrow R$ is a $Q$-algebra and $\omega: T \rightarrow \operatorname{Proj}_{R}$ is a fibre functor, then the stack $\alpha^{*} \mathrm{Fib}(T)$ is isomorphic to $\mathrm{B} G=[\operatorname{Spec}(R) / G]$, where $G$ is the affine group scheme $\underline{\operatorname{Aut}}^{\otimes}(\omega)$ over $R$. This observation (together with the fact that such fibre functors exist) makes $\operatorname{Fib}(T)$ into a gerbe.

A representation of $\operatorname{Fib}(T)$ is a cartesian functor $\operatorname{Fib}(T) \rightarrow$ Proj, in other words, a collection of functors $\operatorname{Fib}(T)_{R} \rightarrow \operatorname{Proj}_{R}$ that is functorial in $R$. The category of representations of $\operatorname{Fib}(T)$ 


\section{MTC FOR PRODUCTS OF ABELIAN VARIETIES}

is denoted by $\operatorname{Rep}(\operatorname{Fib}(T))$, and the evaluation functor $T \rightarrow \operatorname{Rep}(\operatorname{Fib}(T))$, given by $V \mapsto(\omega \mapsto$ $\omega(V)$ ), is an equivalence. This is one half of the statement of Tannaka duality. The other half is the converse statement: if $G$ is an affine gerbe over $Q$, then $G$ is naturally isomorphic to $\operatorname{Fib}(\operatorname{Rep}(G))$.

Definition 2.4. Assume that $T$ is finitely generated (hence generated by one object). The adjoint object in $T$ is the object (well-defined up to isomorphism) that corresponds to the collection of functors $\operatorname{Fib}(T)_{R} \rightarrow \operatorname{Proj}_{R}$ given by $\omega \mapsto \operatorname{Lie}\left(\underline{\operatorname{Aut}}^{\otimes}(\omega)\right)$ via the Tannakian formalism described above. Observe that since $T$ is finitely generated, the group scheme $\underline{\mathrm{Aut}}^{\otimes}(\omega)$ is of finite type, and therefore Lie $\left(\underline{\mathrm{Aut}}^{\otimes}(\omega)\right)$ is finitely generated.

Notation 2.5. If $V$ is an object of $T$, then $V^{\text {ad }}$ denotes the adjoint object of the Tannakian subcategory $\langle V\rangle^{\otimes} \subset T$ generated by $V$.

2.6. If $V$ is an object in $T$, inductively define a sequence of objects by $V^{(0)}=V$ and $V^{(i+1)}=$ $\left(V^{(i)}\right)^{\text {ad }}$ for $i \in \mathbb{Z}_{\geq 0}$. Observe that for $i \geq 1$, the object $V^{(i+1)}$ is a quotient of $V^{(i)}$, and therefore $\operatorname{dim} V^{(i+1)} \leq \operatorname{dim} V^{(i)}$. Since $V$ is finite-dimensional, this sequence stabilises at an object $V^{(\infty)}$.

Definition 2.7. Retain the notation of the preceding paragraph. We call the object $V^{(\infty)}$ the hyperadjoint object associated with $V$, and we denote it by $V^{\text {ha }}$. We say that an object $V \in T$ is hyperadjoint if $V \cong V^{\text {ha }}$ (or, equivalently, if $V \cong V^{\text {ad }}$ ).

Remark 2.8. The constructions $V \leadsto V^{\text {ad }}$ and $V \leadsto V^{\text {ha }}$ are not functorial. They do not in general commute with tensor functors between Tannakian categories. Also, the constructions are not in general compatible with direct sums. Note that the definitions are such that if $V \neq 0$ is a hyperadjoint object in $T$, then $V \oplus V$ is not hyperadjoint. Caveat emptor!

On a more positive note, the following remark explains that in this paper, these constructions are very manageable. Lemma 2.11 also lists some natural properties of these constructions.

Remark 2.9. In this paper, we always have $V^{\text {ha }}=V^{(2)}$ for all objects that are of interest to us. The reason for this is that all the objects we encounter live in Tannakian (sub)categories that are semisimple, and therefore the associated groups (or gerbes) are reductive. Now suppose that $G$ is a reductive group, with a faithful representation $V \in \operatorname{Rep}(G)$. After the first step, we have the object $V^{(1)}=V^{\text {ad }}=\operatorname{Lie}(G)$. Since $G$ is reductive, we have a short exact sequence $0 \rightarrow \mathrm{Z}(G) \rightarrow G \rightarrow G^{\text {ad }} \rightarrow 0$, and $\operatorname{Lie}(G)=\operatorname{Lie}(\mathrm{Z}(G)) \oplus \operatorname{Lie}\left(G^{\text {ad }}\right)$. Observe that $\operatorname{Lie}(\mathrm{Z}(G))$ is isomorphic to a number of copies of the trivial representation of $G$, and therefore $G^{\text {ad }}$ is the group associated with $V^{(1)}$. We conclude that $V^{(2)}=\operatorname{Lie}\left(G^{\text {ad }}\right)$, which is a faithful representation of $G^{\text {ad }}$, and therefore $V^{\text {ha }}=V^{(2)}$.

Remark 2.10. I do not know of an intrinsic way to define adjoint and hyperadjoint objects in a finitely generated Tannakian category. Given the universal nature of the adjoint representation, I expect that it is possible to give a definition without using the Tannakian formalism to pass to algebraic groups or gerbes. Such a definition might also lead to intrinsic proofs of several properties, such as those in the following lemma.

LEMma 2.11. Let $V$ be an object of $T$ and $W$ an object of $\langle V\rangle^{\otimes}$.

(i) We have $W^{\text {ad }} \in\left\langle V^{\text {ad }}\right\rangle^{\otimes}$ and $W^{\text {ha }} \in\left\langle V^{\text {ha }}\right\rangle^{\otimes}$.

(ii) If $V$ is a direct sum of hyperadjoint objects, then $\langle V\rangle^{\otimes}$ is semisimple. 


\section{J. COMmelin}

(iii) If $V$ is a direct sum of hyperadjoint objects, and in addition $W$ is hyperadjoint, then $W$ is a direct summand of $V$.

Suppose $V=V_{1} \oplus V_{2}$ with $V_{1}, V_{2} \in T$.

(iv) The adjoint object $V^{\text {ad }}$ is a subobject of $V_{1}^{\text {ad }} \oplus V_{2}^{\text {ad }}$, and in particular an object of $\left\langle V_{1}^{\text {ad }} \oplus\right.$ $\left.V_{2}^{\text {ad }}\right\rangle^{\otimes}$

(v) For all $i \in \mathbb{Z}_{\geq 0}$, we have $V^{(i+1)} \in\left\langle\left(V_{1}^{(i)} \oplus V_{2}^{(i)}\right)^{\text {ad }}\right\rangle^{\otimes} \subset\left\langle V_{1}^{(i+1)} \oplus V_{2}^{(i+1)}\right\rangle^{\otimes}$.

(vi) The object $V^{\text {ha }}$ is a direct summand of $V_{1}^{\text {ha }} \oplus V_{2}^{\text {ha }}$.

Proof. We give the proof under the assumption that there is a fibre functor $\omega \in \operatorname{Fib}(T)_{Q}$. Let $G$ be the group $\underline{\operatorname{Aut}}^{\otimes}\left(\left.\omega\right|_{\langle V\rangle^{\otimes}}\right)$, and denote the group $\underline{\operatorname{Aut}}^{\otimes}\left(\left.\omega\right|_{\langle W\rangle^{\otimes}}\right)$ by $H$. By assumption, there is a surjective map $G \rightarrow H$.

(i) Since $G$ and $H$ are groups over the field $Q$ of characteristic 0 , the map $G \rightarrow H$ induces a surjection $\operatorname{Lie}(G) \rightarrow \operatorname{Lie}(H)$. This proves the first claim; the second follows by induction.

(ii) If $V$ is hyperadjoint, then $G$ is semisimple and thus reductive. The general case - where $V$ is a direct sum of hyperadjoint objects - follows from Goursat's lemma in the context of algebraic groups (see Remark 1.3(ii)).

(iii) Both $G$ and $H$ are adjoint semisimple, and $H$ is a quotient of $G$. Thus $H$ is a factor of $G$.

Let $G_{i}$ be the group $\underline{\mathrm{Aut}}^{\otimes}\left(\left.\omega\right|_{\left\langle V_{i}\right\rangle^{\otimes}}\right)$. There is a natural map $G \hookrightarrow G_{1} \times G_{2}$, and its composition with the projection onto $G_{1}$ or $G_{2}$ is surjective.

(iv) There is a natural map $\operatorname{Lie}(G) \hookrightarrow \operatorname{Lie}\left(G_{1}\right) \oplus \operatorname{Lie}\left(G_{2}\right)$.

(v) Inductively apply points (i) and (iv).

(vi) Apply points (v) and (iii).

\section{Fractional Hodge structures}

Following [Del79], we use the notion of fractional Hodge structures. We also define the slightly more general notion of a fractional pre-Hodge structure. This section does not contain anything original but only introduces these concepts because they will prove useful in understanding Deligne's construction (Section 8).

Definition 3.1. Let $R \subset \mathbb{R}$ be a subring (typically $\mathbb{Z}, \mathbb{Q}$, or $\mathbb{R}$ ). A fractional pre-Hodge structure over $R$ consists of a free $R$-module $V$ of finite rank and a decomposition $V \otimes \mathbb{C} \cong \bigoplus_{p, q \in \mathbb{Q}} V^{p, q}$ over $\mathbb{C}$ such that $V^{p, q}=\overline{V^{q, p}}$.

We denote the category of fractional pre-Hodge structures over $R$ by $\mathrm{FpHS}_{R}$.

3.2. Let $V$ be a fractional pre-Hodge structure over a $\operatorname{ring} R \subset \mathbb{R}$. For $p, q \in \mathbb{Q}$, we denote the dimension of $V^{p, q}$ by $h^{p, q}(V)$. We say that $V$ is pure of weight $n \in \mathbb{Q}$ if $h^{p, q}(V) \neq 0$ implies $p+q=n$. A fractional Hodge structure is a fractional pre-Hodge structure that is the direct sum of pure fractional pre-Hodge structures. A pre-Hodge structure $V$ (without the adjective fractional) is a fractional pre-Hodge structure for which $h^{p, q}(V) \neq 0$ implies $p, q \in \mathbb{Z}$. If $V$ is both a fractional Hodge structure and a pre-Hodge structure, then $V$ is a Hodge structure in the classical sense of the word. 


\section{MTC FOR PRODUCTS OF ABELIAN VARIETIES}

3.3. Let $\mathbb{S}$ denote the Deligne torus $\operatorname{Res}_{\mathbb{C} / \mathbb{R}} \mathbb{G}_{\mathrm{m}}$. Recall that a Hodge structure over $R$ is completely described by a representation $h: \mathbb{S} \rightarrow \mathrm{GL}(V)_{\mathbb{R}}$, as follows: for $z \in \mathbb{S}(\mathbb{C})$ and $v \in V^{p, q}$, we put $h(z) \cdot v=z^{-p} \bar{z}^{-q} v$. Composing $h$ with the map $x \mapsto x^{k}: \mathbb{S} \rightarrow \mathbb{S}$ amounts to relabeling $V^{p, q}$ as $V^{k p, k q}$.

Put $\tilde{\mathbb{S}}=\lim _{\mathbb{N}} \mathbb{S}$, where $\mathbb{N}$ is ordered by divisibility and for $m \mid n$, we take the transition map $\mathbb{S} \rightarrow \mathbb{S}$ given by $x \mapsto x^{n / m}$. Then $\tilde{\mathbb{S}}$ is a pro-algebraic group scheme, and the category of fractional pre-Hodge structures over $\mathbb{R}$ is equivalent to $\operatorname{Rep}(\tilde{\mathbb{S}})$.

Definition 3.4. Let $V$ be a fractional pre-Hodge structure over a ring $R \subset \mathbb{R}$. The Mumford-Tate group of $V$ is the smallest algebraic subgroup $\mathrm{G}_{\mathrm{B}}(V) \subset \mathrm{GL}(V)$ over $R$ such that $h: \tilde{\mathbb{S}} \rightarrow \mathrm{GL}(V)_{\mathbb{R}}$ factors through $\mathrm{G}_{\mathrm{B}}(V)_{\mathbb{R}} \subset \mathrm{GL}(V)_{\mathbb{R}}$.

Alternatively, let $\omega: \mathrm{FpHS}_{R} \rightarrow \operatorname{Proj}_{R}$ be the forgetful functor. Then $\omega$ is a fibre functor, and $\mathrm{G}_{\mathrm{B}}(V)=\underline{\mathrm{Aut}^{\otimes}}\left(\left.\omega\right|_{\langle V\rangle^{\otimes}}\right)$.

3.5. Let $V$ be a pre-Hodge structure over a $\operatorname{ring} R \subset \mathbb{R}$. Recall that this pre-Hodge structure is described by a morphism $h: \mathbb{S} \rightarrow \mathrm{G}_{\mathrm{B}}(V)_{\mathbb{R}}$. Denote by $\mu_{0}$ the cocharacter $\mathbb{G}_{\mathrm{m}, \mathbb{C}} \rightarrow \mathbb{S}_{\mathbb{C}}$ given by $z \mapsto(z, 1)$ on $\mathbb{C}$-valued points. The composite morphism $\mu_{h}=h_{\mathbb{C}} \circ \mu_{0}$ is called the Hodge cocharacter of $V$. If there is no cause for confusion, we will write $\mu$ for $\mu_{h}$.

Lemma 3.6. Let $V$ be a fractional pre-Hodge structure over $\mathbb{Q}$. The Mumford-Tate group of $V$ is a torus if and only if $V$ is a free module of rank 1 over a commutative semisimple algebra $E \subset \operatorname{End}_{\mathrm{FpHS}_{\mathbb{Q}}}(V)$.

Proof. Assume that $\mathrm{G}_{\mathrm{B}}(V)$ is a torus. Let $T \subset \mathrm{GL}(V)$ be a maximal torus containing $\mathrm{G}_{\mathrm{B}}(V)$. Then $E=\operatorname{End}_{\text {Vect }_{\mathbb{Q}}}(V)^{T} \subset \operatorname{End}_{\text {Vect }_{\mathbb{Q}}}(V)^{\mathrm{G}_{\mathrm{B}}(V)}=\operatorname{End}_{\mathrm{FpHS}_{\mathbb{Q}}}(V)$ is a commutative semisimple algebra, and $V$ has rank 1 over $E$.

Conversely, suppose that $V$ is free of rank 1 over some commutative semisimple algebra $E \subset \operatorname{End}_{\mathrm{FpHS}_{\mathbb{Q}}}(V)$. Then $\mathrm{G}_{\mathrm{B}}(V) \subset \operatorname{Res}_{E / \mathbb{Q}} \mathbb{G}_{\mathrm{m}}=\operatorname{Res}_{E / \mathbb{Q}} \mathrm{GL}_{E}(V)$ is a torus.

Definition 3.7. A fractional pre-Hodge structure $V$ over a ring $R \subset \mathbb{R}$ is called of CM type (or a fractional CM pre-Hodge structure) if the Mumford-Tate group $\mathrm{G}_{\mathrm{B}}(V)$ is a torus.

Lemma 3.8. The full subcategory of $\mathrm{FpHS}_{\mathbb{Q}}$ consisting of (classical) CM Hodge structures over $\mathbb{Q}$ is a Tannakian subcategory generated by Hodge structures of the form $\mathrm{H}_{\mathrm{B}}^{1}(A)$, where $A$ is a complex abelian variety of $\mathrm{CM}$ type.

Proof. See $[$ And92, §2].

\section{Abelian motives}

To give ourselves access to the strength and flexibility of representation theory, we linearise the category of abelian varieties, yielding a category of so-called abelian motives. As a consequence of work of Deligne and André, this category is very tractable (see Theorem 4.13).

4.1. The category of (pure) motives developed by André [And96b] is very suitable for the problems at hand. Alternatively, we could use motives for absolute Hodge cycles; this would not influence the statements of results or proofs. In the next subsection, we recall André's definition. 


\section{J. Commelin}

4.2. Let $K$ be a subfield of $\mathbb{C}$, and let $X$ be a smooth projective variety over $K$. A class $\gamma$ in $\mathrm{H}_{\mathrm{B}}^{2 i}(X)$ is called a motivated cycle of degree $i$ if there exists an auxiliary smooth projective variety $Y$ over $K$ such that $\gamma$ is of the form $\pi_{*}(\alpha \cup \star \beta)$, where $\pi: X \times Y \rightarrow X$ is the projection, $\alpha$ and $\beta$ are algebraic cycle classes in $\mathrm{H}_{\mathrm{B}}^{*}(X \times Y)$, and $\star \beta$ is the image of $\beta$ under the Hodge star operation. (Alternatively, one may use the Lefschetz star operation; see [And96b, §1].)

Every algebraic cycle is motivated, and under the Lefschetz standard conjecture, the converse holds as well. The set of motivated cycles naturally forms a graded $\mathbb{Q}$-algebra. The category of motives over $K$, denoted by $\operatorname{Mot}_{K}$, consists of objects $(X, p, m)$, where $X$ is a smooth projective variety over $K, p$ is an idempotent motivated cycle on $X \times X$, and $m$ is an integer. A morphism $(X, p, m) \rightarrow(Y, q, n)$ is a motivated cycle $\gamma$ of degree $n-m$ on $Y \times X$ such that $q \gamma p=\gamma$. We denote the object $(X, \Delta, 0)$, where $\Delta$ is the class of the diagonal in $X \times X$, by $\mathrm{H}(X)$. The Künneth projectors $\pi_{i}$ are motivated cycles, and we denote the object $\left(X, \pi_{i}, 0\right)$ by $\mathrm{H}^{i}(X)$. Observe that $\mathrm{H}(X)=\oplus_{i} \mathrm{H}^{i}(X)$. This gives contravariant functors $\mathrm{H}\left(\_\right)$and $\mathrm{H}^{i}\left(\_\right)$from the category of smooth projective varieties over $K$ to $\operatorname{Mot}_{K}$.

Theorem 4.3. The category $\operatorname{Mot}_{K}$ is Tannakian over $\mathbb{Q}$, semisimple, graded, and polarised. Every classical cohomology theory of smooth projective varieties over $K$ factors via $\operatorname{Mot}_{K}$.

Proof. See [And96b, théorème 0.4].

4.4. As Theorem 4.3 indicates, the category of motives that we use is designed to have realisation functors for all the classical cohomology theories. For each prime number $\ell$, we obtain an $\ell$-adic realisation functor $\mathrm{H}_{\ell}: \operatorname{Mot}_{K} \rightarrow \operatorname{Rep}_{\mathbb{Q}_{\ell}}\left(\Gamma_{K}\right)$ to $\ell$-adic Galois representations of $K$, and we obtain a Betti-realisation functor $\mathrm{H}_{\mathrm{B}}: \operatorname{Mot}_{K} \rightarrow \mathrm{HS}_{\mathbb{Q}}$ to the category of Hodge structures over $\mathbb{Q}$. There is a natural isomorphism $\mathrm{H}_{\ell}\left(\_\right) \cong \mathrm{H}_{\mathrm{B}}\left(\_\right) \otimes \mathbb{Q}_{\ell}$ of functors from $\operatorname{Mot}_{K}$ to $\mathbb{Q}_{\ell}$-vector spaces.

Definition 4.5. Let $K$ be a subfield of $\mathbb{C}$. The motivic Galois group $\mathrm{G}\left(\mathrm{Mot}_{K}\right)$ is the proalgebraic affine group scheme $\underline{\mathrm{Aut}}^{\otimes}\left(\mathrm{H}_{\mathrm{B}}\right)$ over $\mathbb{Q}$ associated with $\mathrm{Mot}_{K}$ via the Tannakian formalism. If $M$ is a motive over $K$, then we denote the affine group scheme associated with $\langle M\rangle^{\otimes} \subset \operatorname{Mot}_{K}$ by $\mathrm{G}(M)$; it is the image of $\mathrm{G}\left(\operatorname{Mot}_{K}\right)$ in $\mathrm{GL}\left(\mathrm{H}_{\mathrm{B}}(M)\right)$.

4.6. If $K \subset L$ is an extension of subfields of $\mathbb{C}$, then there is a natural functor $\operatorname{Mot}_{K} \rightarrow \operatorname{Mot}_{L}$. If $K$ and $L$ are algebraically closed, this functor is fully faithful. If $L$ is algebraic over $K$, there is a short exact sequence $1 \rightarrow \mathrm{G}\left(\operatorname{Mot}_{L}\right) \rightarrow \mathrm{G}\left(\operatorname{Mot}_{K}\right) \rightarrow \mathrm{Gal}(L / K) \rightarrow 1$. (Via the Tannakian formalism, the Galois group $\operatorname{Gal}(L / K)$ corresponds to the subcategory of $\operatorname{Mot}_{K}$ generated by the objects $\mathrm{H}\left(\operatorname{Spec}\left(K^{\prime}\right)\right)$, where $K^{\prime}$ is an intermediate field $K \subset K^{\prime} \subset L$.)

It is not known whether $\mathrm{G}\left(\operatorname{Mot}_{\bar{K}}\right)$ is connected.

Notation 4.7. Let $M$ be a motive over a field $K \subset \mathbb{C}$. We denote the Zariski closure of the image of the Galois representation $\Gamma_{K} \rightarrow \mathrm{GL}\left(\mathrm{H}_{\ell}(M)\right)$ by $\mathrm{G}_{\ell}(M)$, and we write $\mathrm{G}_{\ell}^{\circ}(M)$ for the identity component of $\mathrm{G}_{\ell}(M)$. We denote the Mumford-Tate group of the Hodge structure $\mathrm{H}_{\mathrm{B}}(M)$ by $\mathrm{G}_{\mathrm{B}}(M)$. The realisation functors induce natural injective morphisms $\mathrm{G}_{\ell}(M) \rightarrow \mathrm{G}(M)_{\mathbb{Q}_{\ell}}$ (via the comparison isomorphism) and $\mathrm{G}_{\mathrm{B}}(M) \rightarrow \mathrm{G}(M)$.

Remark 4.8. In certain situations, it is expected that the natural morphisms mentioned above are isomorphisms. Let us make this more precise. Let $M$ be a motive over a field $K \subset \mathbb{C}$. Assume that $K$ is finitely generated. The Tate conjecture predicts that invariants of $\mathrm{G}_{\ell}(M)$ (in tensor powers of $\left.\mathrm{H}_{\ell}(M)\right)$ are algebraic and, in particular, motivated. Suppose that $\mathrm{G}_{\ell}(M)$ is reductive, so that it is determined by the invariant subspace in the tensor algebra on $\mathrm{H}_{\ell}(M)$. If the invariants 
of $\mathrm{G}_{\ell}(M)$ and $\mathrm{G}(M)_{\mathbb{Q}_{\ell}}$ agree, then the two groups are isomorphic. (In fact, we do not need to assume that $\mathrm{G}_{\ell}(M)$ is reductive, by the result of [Moo19].)

Now assume $K=\mathbb{C}$. The Hodge conjecture predicts that invariants of $\mathrm{G}_{\mathrm{B}}(M)$ in the tensor algebra on $\mathrm{H}_{\mathrm{B}}(M)$ are algebraic and thus motivated. We already know that $\mathrm{G}_{\mathrm{B}}(M)$ is reductive, and hence the Hodge conjecture predicts that $\mathrm{G}_{\mathrm{B}}(M) \rightarrow \mathrm{G}(M)$ is an isomorphism. See Theorem 4.13 for an example of a class of motives where we know "Hodge = motivated".

The Tate and Hodge conjectures are naturally complemented by a third conjecture: the Mumford-Tate conjecture, that we state below. We leave it to the reader to verify that if any two of the three conjectures hold (for all motives), then so does the third.

Conjecture 4.9. Let $M$ be a motive over a finitely generated field $K \subset \mathbb{C}$. Fix a prime number $\ell$. The Mumford-Tate conjecture $\operatorname{MTC}_{\ell}(M)$ for $M$ is the statement that under the comparison isomorphism $\mathrm{H}_{\ell}(M) \cong \mathrm{H}_{\mathrm{B}}(M) \otimes \mathbb{Q}_{\ell}$, we have

$$
\mathrm{G}_{\ell}^{\circ}(M) \cong \mathrm{G}_{\mathrm{B}}(M) \otimes \mathbb{Q}_{\ell} .
$$

We write $\operatorname{MTC}(M)$ for the conjecture $\forall \ell: \operatorname{MTC}_{\ell}(M)$.

Remark 4.10. (1) Let $M$ be a motive over a field $K \subset \mathbb{C}$. By definition, there is a smooth projective variety $X$ such that $M \in\langle\mathrm{H}(X)\rangle^{\otimes}$. By work of Serre [Ser81], there is a finite extension $L / K$ such that $\mathrm{G}_{\ell}\left(\mathrm{H}\left(X_{L}\right)\right)$ is connected for all $\ell$. Since $M \in\langle\mathrm{H}(X)\rangle^{\otimes}$, there is a quotient map $\mathrm{G}_{\ell}\left(\mathrm{H}\left(X_{L}\right)\right) \rightarrow \mathrm{G}_{\ell}\left(M_{L}\right)$, and therefore $\mathrm{G}_{\ell}\left(M_{L}\right)$ is connected for all $\ell$.

(2) If $L / K$ is a finitely generated extension field, then there is an isomorphism $\mathrm{G}_{\ell}^{\circ}\left(M_{L}\right) \cong \mathrm{G}_{\ell}^{\circ}(M)$ (see [Moo17b, Proposition 1.3]). Therefore, the Mumford-Tate conjecture for $M$ is equivalent to the Mumford-Tate conjecture for $M_{L}$. In particular, when trying to prove this conjecture for $M$, we may always assume that $\mathrm{G}_{\ell}(M)$ is connected for all prime numbers $\ell$.

Definition 4.11. (i) An Artin motive over a field $K \subset \mathbb{C}$ is an object in the Tannakian subcategory of $\operatorname{Mot}_{K}$ generated by motives of the form $\mathrm{H}(\operatorname{Spec}(L))$, where $L$ is a finite field extension of $K$.

(ii) An abelian motive over $K$ is an object in the Tannakian subcategory of $\operatorname{Mot}_{K}$ generated by Artin motives and motives of the form $\mathrm{H}(A)$, where $A$ is an abelian variety over $K$.

Remark 4.12. In practice, we can ignore Artin motives in this paper. The Mumford-Tate conjecture is trivially true for them: if $M$ is an Artin motive, then both $\mathrm{G}_{\mathrm{B}}(M)$ and $\mathrm{G}_{\ell}^{\circ}(M)$ are trivial. (Note that $\mathrm{G}_{\ell}(M)$ can be a non-trivial finite group.)

If $M$ is an arbitrary abelian motive, then there are always a finite field extension $L / K$ and an abelian variety $A / L$ such that $M_{L} \in\langle A\rangle^{\otimes} \subset \operatorname{Mot}_{L}$. By Remark 4.10(ii), we know that MTC $(M)$ is equivalent to $\operatorname{MTC}\left(M_{L}\right)$, and therefore we may restrict our attention to motives in the Tannakian subcategory generated by abelian varieties over $K$.

Theorem 4.13. Consider the category of motives over $\mathbb{C}$. The restriction of the Hodge realisation functor $\mathrm{H}_{\mathrm{B}}\left(\_\right)$to the subcategory of abelian motives is a full functor.

Proof. See [And96b, théorème 0.6.2].

Remark 4.14. (1) Let $M$ be an abelian motive over a field $K \subset \mathbb{C}$. A corollary of Theorem 4.13 is that $\mathrm{G}_{\mathrm{B}}(M)$ is the identity component of $\mathrm{G}(M)$. In particular, we obtain the inclusion $\mathrm{G}_{\ell}^{\circ}(M) \subset$ $\mathrm{G}_{\mathrm{B}}(M) \otimes \mathbb{Q}_{\ell}$. 


\section{J. Commelin}

(2) Let $A$ be an abelian variety over $K$, and assume that $K$ is finitely generated. Then $\mathrm{G}_{\ell}^{\circ}(A)$ is a reductive group by $[$ Fal83, $\S 5$, Satz 3]. (See also [Fal84] for the case where $K$ is not a number field.) Suppose $M \in\left\langle\mathrm{H}^{1}(A)\right\rangle^{\otimes}$. Then there is a surjection $\mathrm{G}_{\ell}^{\circ}(A) \rightarrow \mathrm{G}_{\ell}^{\circ}(M)$. Hence $\mathrm{G}_{\ell}^{\circ}(M)$ is reductive for every abelian motive $M$.

(3) We also know that $\operatorname{End}_{\mathrm{G}_{\ell}^{\circ}(A)}\left(\mathrm{H}_{\ell}^{1}(A)\right) \cong \operatorname{End}_{\mathrm{G}_{\mathrm{B}}(A)}\left(\mathrm{H}_{\mathrm{B}}^{1}(A)\right)_{\mathbb{Q}_{\ell}}$, by [Fal83, §5, Satz 4] (see also [Fal84]). Now observe that $\mathrm{G}_{\ell}^{\circ}(A)$ embeds into End $_{\text {Vect }}\left(\mathrm{H}_{\ell}^{1}(A)\right)$, and by the definition of the centre, we have $\mathrm{Z}\left(\mathrm{G}_{\ell}^{\circ}(A)\right)=\mathrm{G}_{\ell}^{\circ}(A) \cap \operatorname{End}_{\mathrm{G}_{\ell}^{\circ}(A)}\left(\mathrm{H}_{\ell}^{1}(A)\right)$, where the intersection takes place inside $\operatorname{End}_{\text {Vect }}\left(\mathrm{H}_{\ell}^{1}(A)\right)$. Similarly, we have $\mathrm{Z}\left(\mathrm{G}_{\mathrm{B}}(A)\right)_{\mathbb{Q}_{\ell}}=\mathrm{G}_{\mathrm{B}}(A)_{\mathbb{Q}_{\ell}} \cap \operatorname{End}_{\mathrm{G}_{\mathrm{B}}(A)}\left(\mathrm{H}_{\mathrm{B}}^{1}(A)\right)_{\mathbb{Q}_{\ell}}$. With the two preceding points in mind, we conclude that $\mathrm{Z}\left(\mathrm{G}_{\ell}^{\circ}(A)\right) \subset \mathrm{Z}\left(\mathrm{G}_{\mathrm{B}}(A)\right)_{\mathbb{Q}_{\ell}}$.

Lemma 4.15. Let $M$ be an abelian motive over a finitely generated field $K \subset \mathbb{C}$. Fix a prime number $\ell$. Under the comparison isomorphism $\mathrm{H}_{\ell}(M)=\mathrm{H}_{\mathrm{B}}(M) \otimes \mathbb{Q}_{\ell}$, we have an inclusion of centres $\mathrm{Z}\left(\mathrm{G}_{\ell}^{\circ}(M)\right) \subset \mathrm{Z}\left(\mathrm{G}_{\mathrm{B}}(M)\right)_{\mathbb{Q}_{\ell}}$.

Proof. After replacing $K$ with a finite field extension $L / K$, there is an abelian variety $A / L$ such that $M_{L} \in\left\langle\mathrm{H}^{1}(A)\right\rangle^{\otimes}$ (see Remark 4.12). By Remark 4.10(ii), we have $\mathrm{G}_{\ell}^{\circ}\left(M_{L}\right)=\mathrm{G}_{\ell}^{\circ}(M)$, and hence we may and do assume $L=K$. Now we have a commutative diagram

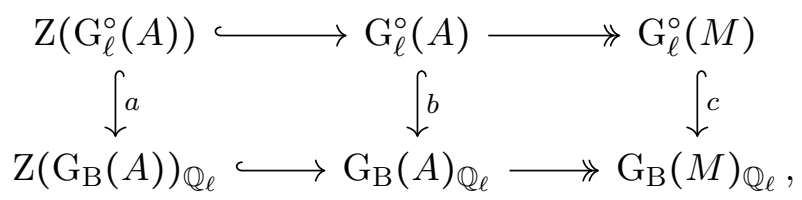

where $b$ and $c$ exist by Theorem 4.13 (see Remark 4.14(i)) and $a$ exists by Remark 4.14(iii). (Note: we do not claim that the rows are exact.)

Since $\mathrm{G}_{\ell}^{\circ}(A)$ and $\mathrm{G}_{\ell}^{\circ}(M)$ are reductive (see Remark 4.14(ii)), the image of $\mathrm{Z}\left(\mathrm{G}_{\ell}^{\circ}(A)\right.$ ) by the quotient map to $\mathrm{G}_{\ell}^{\circ}(M)$ is exactly $\mathrm{Z}\left(\mathrm{G}_{\ell}^{\circ}(M)\right)$. Similarly, the image of $\mathrm{Z}\left(\mathrm{G}_{\mathrm{B}}(A)\right)_{\mathbb{Q}_{\ell}}$ in $\mathrm{G}_{\mathrm{B}}(M)_{\mathbb{Q}_{\ell}}$ is $\mathrm{Z}\left(\mathrm{G}_{\mathrm{B}}(M)\right)_{\mathbb{Q}_{\ell}}$. The result follows from the commutativity of the above diagram.

4.16. Let $M$ be a motive over a field $K \subset \mathbb{C}$. In this article, we say that $M$ is of $\mathrm{CM}$ type (or a $\mathrm{CM}$ motive) if the identity component $\mathrm{G}^{\circ}(M)$ of the motivic Galois group $\mathrm{G}(M)$ is a torus. The full subcategory of $\operatorname{Mot}_{K}$ consisting of abelian CM motives is a Tannakian subcategory generated by Artin motives and motives of the form $\mathrm{H}^{1}(A)$, where $A$ is a $\mathrm{CM}$ abelian variety over $K$ (cf. Lemma 3.8).

Lemma 4.17. Let $M$ be an abelian CM motive over a finitely generated field $K \subset \mathbb{C}$. Then the Mumford-Tate conjecture is true for $M$.

Proof. After we replace $K$ with a finite field extension $L / K$, there is a CM abelian variety $A / L$ such that $M_{L} \in\left\langle\mathrm{H}^{1}(A)\right\rangle^{\otimes}$ (see Remark 4.12). By [Poh68], the Mumford-Tate conjecture is true for $A$, and thus $\operatorname{MTC}(M)$ is true by Remark 4.10(ii).

Lemma 4.18. Let $M$ be an abelian motive over a finitely generated field $K \subset \mathbb{C}$. Fix a prime number $\ell$. Under the comparison isomorphism $\mathrm{H}_{\ell}(M)=\mathrm{H}_{\mathrm{B}}(M) \otimes \mathbb{Q}_{\ell}$, we have an inclusion of centres $\mathrm{Z}\left(\mathrm{G}_{\ell}^{\circ}(M)\right) \subset \mathrm{Z}\left(\mathrm{G}_{\mathrm{B}}(M)\right) \otimes \mathbb{Q}_{\ell}$, and $\mathrm{Z}\left(\mathrm{G}_{\ell}^{\circ}(M)\right)^{\circ}=\mathrm{Z}\left(\mathrm{G}_{\mathrm{B}}(M)\right)^{\circ} \otimes \mathbb{Q}_{\ell}$.

Proof. (This result is proven in [Vas08, Theorem 1.3.1] and [UY13, Corollary 2.11] using different methods.) The first claim is Lemma 4.15. Therefore, it suffices to show that the composition of the natural maps $\mathrm{G}_{\ell}^{\circ}(M) \hookrightarrow \mathrm{G}_{\mathrm{B}}(M)_{\mathbb{Q}_{\ell}} \rightarrow \mathrm{G}_{\mathrm{B}}(M)_{\mathbb{Q}_{\ell}}^{\text {ab }}$ is surjective. 
Let $N$ be a faithful representation of $\mathrm{G}_{\mathrm{B}}(M)^{\mathrm{ab}}$. By Theorem 4.13 and the Tannakian formalism, we may view $N$ as a complex motive. Note that $N$ is an abelian CM motive, by construction. After replacing $K$ by a finitely generated extension — which is harmless by Remark 4.10(ii) —we may assume that $N$ is defined over $K$. Since the Mumford-Tate conjecture holds for CM motives (Lemma 4.17), we find $\mathrm{G}_{\ell}^{\circ}(N)=\mathrm{G}_{\mathrm{B}}(N)_{\mathbb{Q}_{\ell}}=\mathrm{G}_{\mathrm{B}}(M)_{\mathbb{Q}_{\ell}}^{\mathrm{ab}}$. To complete the proof, we remark that $\mathrm{G}_{\ell}^{\circ}(N)$ is exactly the image of $\mathrm{G}_{\ell}^{\circ}(M)$ under the composite map of the preceding paragraph.

Proposition 4.19. Let $M$ be an abelian motive over a finitely generated field $K \subset \mathbb{C}$. Fix a prime number $\ell$. Then $\operatorname{MTC}_{\ell}(M)$ is equivalent to $\operatorname{MTC}_{\ell}\left(M^{\text {ha }}\right)$, where $M^{\text {ha }}$ is the hyperadjoint object associated with $M \in \operatorname{Mot}_{K}$; see Definition 2.7.

Proof. Consider the commutative diagram

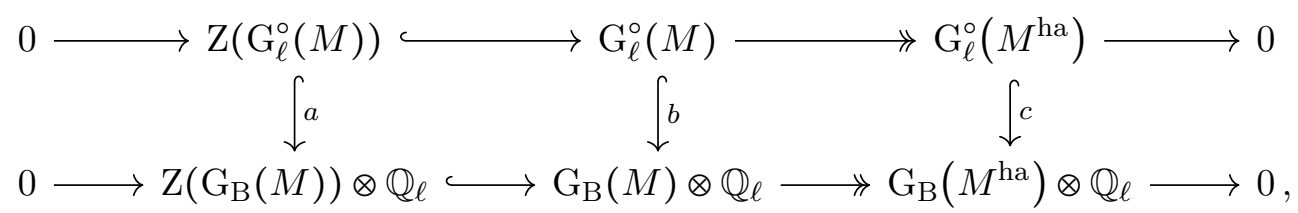

where the inclusions $b$ and $c$ exist by Theorem 4.13 (see Remark 4.14(i)) and $a$ exists by Lemma 4.15. Moreover, $a$ is an isomorphism on identity components, by Lemma 4.18. The bottom row is exact by the definition of hyperadjoint objects (Definition 2.7) and Theorem 4.13.

Certainly, if $b$ is an isomorphism, then $c$ is also an isomorphism. Conversely, if $c$ is an isomorphism, then $\operatorname{dim} \mathrm{G}_{\ell}^{\circ}(M)=\operatorname{dim} \mathrm{G}_{\mathrm{B}}(M)$. Since $b$ is an inclusion of connected linear groups, it must be an isomorphism.

\section{Quasi-compatible systems of Galois representations}

In general, it is expected that the $\ell$-adic realisations $\mathrm{H}_{\ell}(M)$ of a motive $M$ over a finitely generated field $K$ of characteristic 0 form a compatible system of Galois representations in the sense of Serre.

The main theorem of [Com17] shows that if $M$ is an abelian motive, a slightly weaker result is true; this is good enough for our purposes. The weaker condition is called quasi-compatibility and in this section, we recall the necessary definitions and results.

5.1. Let $\kappa$ be a finite field with $q$ elements. We denote by $F_{\kappa} \in \Gamma_{\kappa}$ the geometric Frobenius automorphism, that is, the inverse of $x \mapsto x^{q}$.

Definition 5.2. Let $K$ be a number field. Let $v$ be a finite place of $K$, and let $K_{v}$ denote the completion of $K$ at $v$. Let $\bar{K}_{v}$ be an algebraic closure of $K_{v}$, and let $\bar{\kappa} / \kappa$ be the extension of residue fields corresponding to $\bar{K}_{v} / K_{v}$. The inertia group, denoted by $I_{v}$, is the kernel of the natural surjection $\Gamma_{K_{v}} \rightarrow \Gamma_{\kappa}$. The inverse image of $F_{\kappa}$ in $\Gamma_{K_{v}}$ is called the Frobenius coset of $v$. An element $\alpha \in \Gamma_{K}$ is called a Frobenius element with respect to $v$ if there exists an embedding $\bar{K} \hookrightarrow \bar{K}_{v}$ such that $\alpha$ is the restriction of an element of the Frobenius coset of $v$.

Definition 5.3. Let $K$ be a finitely generated field. A model of $K$ is an integral scheme $X$ of finite type over $\operatorname{Spec}(\mathbb{Z})$ together with an isomorphism between $K$ and the function field of $X$.

Remark 5.4. If $K$ is a number field and $R \subset K$ is an order, then $\operatorname{Spec}(R)$ is naturally a model of $K$. The only model of a number field $K$ that is normal and proper over $\operatorname{Spec}(\mathbb{Z})$ is $\operatorname{Spec}\left(\mathcal{O}_{K}\right)$. 


\section{J. Commelin}

Definition 5.5. Let $K$ be a finitely generated field, and let $X$ be a model of $K$. We denote the set of closed points of $X$ by $X^{\mathrm{cl}}$. Let $x \in X^{\mathrm{cl}}$ be a closed point, let $K_{x}$ be the function field of the Henselisation of $X$ at $x$, and let $\kappa(x)$ be the residue field at $x$. We denote the kernel of $\Gamma_{K_{x}} \rightarrow \Gamma_{\kappa(x)}$ by $I_{x}$. Every embedding $\bar{K} \hookrightarrow \bar{K}_{x}$ induces an inclusion $\Gamma_{K_{x}} \rightarrow \Gamma_{K}$. Like in Definition 5.2, the inverse image of $F_{\kappa(x)}$ in $\Gamma_{K_{x}}$ is called the Frobenius coset of $x$. An element $\alpha \in \Gamma_{K}$ is called a Frobenius element with respect to $x$ if there exists an embedding $\bar{K} \leftrightarrow \bar{K}_{x}$ such that $\alpha$ is the restriction of an element of the Frobenius coset of $x$.

Definition 5.6. Let $K$ be a field, let $E$ be a number field, and let $\lambda$ be a finite place of $E$. A $\lambda$-adic Galois representation of $K$ is a representation of $\Gamma_{K}$ on a finite-dimensional $E_{\lambda}$-vector space $V$ that is continuous for the $\lambda$-adic topology. We denote by $\mathrm{G}_{\lambda}(V) \subset \mathrm{GL}(V)$ the algebraic group over $E_{\lambda}$ that is the Zariski closure of the image of $\Gamma_{K}$ under this representation. The identity component of $\mathrm{G}_{\lambda}(V)$ is denoted by $\mathrm{G}_{\lambda}^{\circ}(V)$.

Definition 5.7. Let $K$ be a field, let $E$ be a number field, and let $\lambda$ be a finite place of $E$. Let $\rho$ be a $\lambda$-adic Galois representation of $K$. Let $X$ be a model of $K$, and let $x \in X^{\text {cl }}$ be a closed point. We say that $\rho$ is unramified at $x$ if there is an embedding $\bar{K} \hookrightarrow \bar{K}_{x}$ for which $\rho\left(I_{x}\right)=\{1\}$, where $I_{x}$ is the kernel of the projection $\Gamma_{K_{x}} \rightarrow \Gamma_{\kappa(x)}$, as in Definition 5.5. (Remark: If this condition is satisfied by one embedding, then it is satisfied by all embeddings.)

Notation 5.8. Let $K, X, x, E, \lambda$, and $\rho$ be as in Definition 5.7, and assume that $K$ is a finitely generated field. Let $F_{x}$ be a Frobenius element with respect to $x$. Assume that $\rho$ is unramified at $x$, so that the element $F_{x, \rho}=\rho\left(F_{x}\right)$ is well defined up to conjugation. For $n \in \mathbb{Z}$, we write $\mathrm{P}_{x, \rho, n}(t)$ for the characteristic polynomial of $F_{x, \rho}^{n}$. Note that $\mathrm{P}_{x, \rho, n}(t)$ does not depend on the choice of $F_{x}$ since conjugate endomorphisms have the same characteristic polynomial.

Definition 5.9. Let $K, X, x, E, \lambda$, and $\rho$ be as in Definition 5.7, and assume that $K$ is a finitely generated field. The representation $\rho$ is said to be E-rational at $x$ if $\rho$ is unramified at $x$ and $\mathrm{P}_{x, \rho, n}(t) \in E[t]$ for some $n \geq 1$.

DeFinition 5.10. Let $K$ be a finitely generated field. Let $E$ be a number field, and let $\lambda_{1}$ and $\lambda_{2}$ be two finite places of $E$. For $i=1,2$, let $\rho_{i}$ be a $\lambda_{i}$-adic Galois representation of $K$.

(i) Let $X$ be a model of $K$, and let $x \in X^{\mathrm{cl}}$ be a closed point. Then $\rho_{1}$ and $\rho_{2}$ are said to be quasi-compatible at $x$ if $\rho_{1}$ and $\rho_{2}$ are both $E$-rational at $x$ and if there is an integer $n$ such that $\mathrm{P}_{x, \rho_{1}, n}(t)=\mathrm{P}_{x, \rho_{2}, n}(t)$ as polynomials in $E[t]$.

(ii) Let $X$ be a model of $K$. The representations $\rho_{1}$ and $\rho_{2}$ are quasi-compatible with respect to $X$ if there is a non-empty open subset $U \subset X$ such that $\rho_{1}$ and $\rho_{2}$ are quasi-compatible at $x$ for all $x \in U^{\mathrm{cl}}$.

(iii) The representations $\rho_{1}$ and $\rho_{2}$ are quasi-compatible if they are quasi-compatible with respect to every model of $K$.

DeFinition 5.11. Let $K$ be a field. By a system of Galois representations of $K$, we mean a triple $\left(E, \Lambda,\left(\rho_{\lambda}\right)_{\lambda \in \Lambda}\right)$, where $E$ is a number field, $\Lambda$ is a set of finite places of $E$, and $\rho_{\lambda}$ (for $\lambda \in \Lambda$ ) is a $\lambda$-adic Galois representation of $K$.

DeFinition 5.12. Let $K$ be a finitely generated field. Let $\rho_{\Lambda}=\left(E, \Lambda,\left(\rho_{\lambda}\right)_{\lambda \in \Lambda}\right)$ be a system of Galois representations of $K$.

(i) Let $X$ be a model of $K$. The system $\rho_{\Lambda}$ is quasi-compatible with respect to $X$ if for all $\lambda_{1}, \lambda_{2} \in \Lambda$, the representations $\rho_{\lambda_{1}}$ and $\rho_{\lambda_{2}}$ are quasi-compatible with respect to $X$. 


\section{MTC FOR PRODUCTS OF ABELIAN VARIETIES}

(ii) The system $\rho_{\Lambda}$ is called quasi-compatible if for all $\lambda_{1}, \lambda_{2} \in \Lambda$, the representations $\rho_{\lambda_{1}}$ and $\rho_{\lambda_{2}}$ are quasi-compatible.

THEOREM 5.13. Let $K$ be a finitely generated field of characteristic 0. Let $M$ be an abelian motive over $K$. Let $E$ be a subfield of $\operatorname{End}(M)$, and let $\Lambda$ be the set of finite places of $E$. Then the system $\mathrm{H}_{\Lambda}(M)$ is a quasi-compatible system of representations.

Proof. See [Com17, Theorem 5.1].

Proposition 5.14. Let $K$ be a finitely generated field. Let $E$ be a number field, and let $\lambda$ be a finite place of $E$. Let $\rho_{1}$ and $\rho_{2}$ be $\lambda$-adic Galois representations of $K$. If $\rho_{1}$ and $\rho_{2}$ are semisimple, quasi-compatible, and $\mathrm{G}_{\lambda}\left(\rho_{1} \oplus \rho_{2}\right)$ is connected, then $\rho_{1} \cong \rho_{2}$.

Proof. See [Com17, Proposition 6.3].

Proposition 5.15. Let $K$ be a finitely generated field. Let $E$ be a number field, and let $\Lambda$ be the set of finite places of $E$ whose residue characteristic is different from char $(K)$. Let $\mathscr{L}$ be the set of prime numbers different from $\operatorname{char}(K)$. Let $\rho_{\Lambda}$ be a quasi-compatible system of representations of $K$. Let $\rho_{\mathscr{L}}$ be the quasi-compatible system of representations obtained from $\rho_{\Lambda}$ by restricting to $\mathbb{Q} \subset E$; in other words, $\rho_{\ell}=\bigoplus_{\lambda \mid \ell} \rho_{\lambda}$. Assume that $\mathrm{G}_{\ell}\left(\rho_{\ell}\right)$ is connected for all $\ell \in \mathscr{L}$. Fix $\lambda_{0} \in \Lambda$. Define the field $E^{\prime} \subset E$ to be the subfield of $E$ generated by elements $e \in E$ that satisfy the following condition:

There exist a model $X$ of $K$, a point $x \in X^{\mathrm{cl}}$, and an integer $n \geq 1$, such that $\mathrm{P}_{x, \rho_{\lambda_{0}}, n}(t) \in$ $E[t]$ and $e$ is a coefficient of $\mathrm{P}_{x, \rho_{\lambda_{0}}, n}(t)$.

Let $\ell$ be a prime number that splits completely in $E / \mathbb{Q}$. If $\operatorname{End}_{\Gamma_{K}, \mathbb{Q}_{\ell}}\left(\rho_{\ell}\right) \cong E \otimes \mathbb{Q}_{\ell}$, then $E=E^{\prime}$.

Proof. We restrict our attention to a finite subset of $\Lambda$, namely $\Lambda_{0}=\left\{\lambda_{0}\right\} \cup\{\lambda \mid \ell\}$. Let $U \subset X$ be a non-empty open subset such that for all $\lambda_{1}, \lambda_{2} \in \Lambda_{0}$, the representations $\rho_{\lambda_{1}}$ and $\rho_{\lambda_{2}}$ are quasicompatible at all $x \in U^{\mathrm{cl}}$. For each $x \in U^{\mathrm{cl}}$, let $n_{x}$ be an integer such that $\mathrm{P}_{x}(t)=\mathrm{P}_{x, \rho_{\lambda}, n_{x}}(t) \in E[t]$ does not depend on $\lambda \in \Lambda_{0}$.

Let $\lambda^{\prime}$ be a place of $E^{\prime}$ above $\ell$. Let $\lambda_{1}$ and $\lambda_{2}$ be two places of $E$ that lie above $\lambda^{\prime}$. We view $\rho_{\lambda_{1}}$ and $\rho_{\lambda_{2}}$ as $\lambda^{\prime}$-adic Galois representation. Since $\ell$ splits completely in $E / \mathbb{Q}$, the inclusions $\mathbb{Q}_{\ell} \subset E_{\lambda^{\prime}}^{\prime} \subset E_{\lambda_{i}}$ are isomorphisms. By the definition of $E^{\prime}$, we have $\mathrm{P}_{x}(t) \in E^{\prime}[t]$. Therefore, $\rho_{\lambda_{1}}$ and $\rho_{\lambda_{2}}$ are quasi-compatible $\lambda^{\prime}$-adic Galois representations; hence, they are isomorphic by Proposition 5.14. Let $\rho_{\lambda^{\prime}}$ be the $\lambda^{\prime}$-adic Galois representation $\oplus_{\lambda \mid \lambda^{\prime}} \rho_{\lambda}$. We conclude that $\operatorname{End}_{\Gamma_{K}, E_{\lambda^{\prime}}^{\prime}}\left(\rho_{\lambda^{\prime}}\right) \cong \operatorname{Mat}_{\left[E: E^{\prime}\right]}\left(E_{\lambda^{\prime}}^{\prime}\right)$, which implies $\left[E: E^{\prime}\right]=1$.

\section{Deligne-Dynkin diagrams}

In order to streamline the discussion of Deligne's construction (Section 8) and the proof of the main theorem in the final two sections, in this section, we attempt to axiomatise parts of [Del79] (notably $\S 1.3$ and $\S 2.3$ ).

We introduce Deligne-Dynkin diagrams: they are Dynkin diagrams with an extra structure that capture important information about hyperadjoint abelian motives. The main result of this section is Proposition 6.23, a local-global result for irreducible symplectic populated DeligneDynkin diagrams over $\mathbb{Q}$.

Definition 6.1. Let $\Delta$ be a connected Dynkin diagram, and let $\Delta^{+}=\Delta \sqcup\left\{\alpha_{0}\right\}$ be the extended (or affine) Dynkin diagram associated with $\Delta$. (See Figure 1.) A node of $\Delta$ is special if it is 


\section{J. COMmelin}

$\mathrm{A}_{1}^{+}: 0<0$

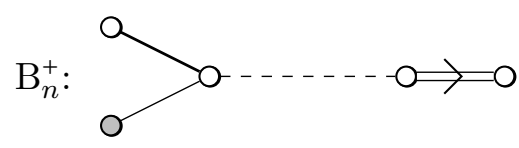

$\mathrm{D}_{n}^{+}:>_{0}^{a}>0$

$\mathrm{F}_{4}^{+}$:

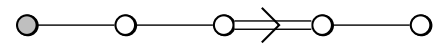

$\mathrm{A}_{n}^{+}(n \geq 2):$
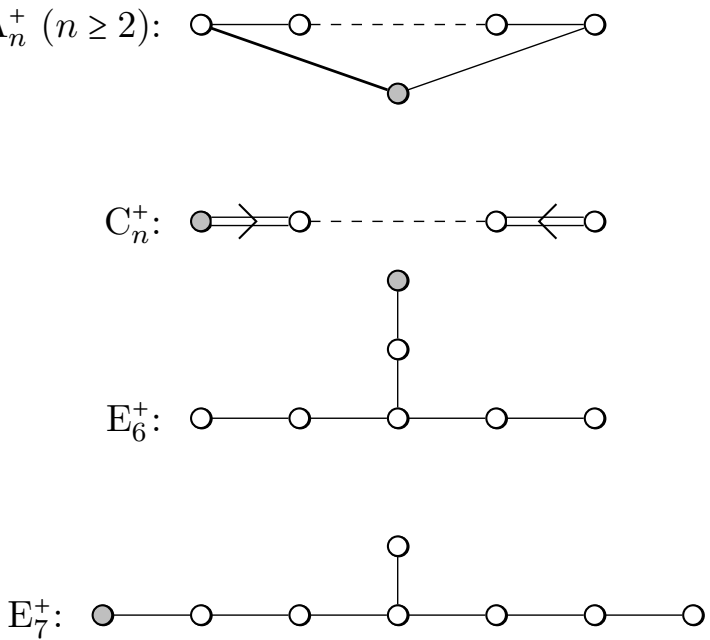

$\mathrm{G}_{2}^{+}$:

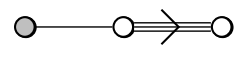

$\mathrm{E}_{8}^{+}$:

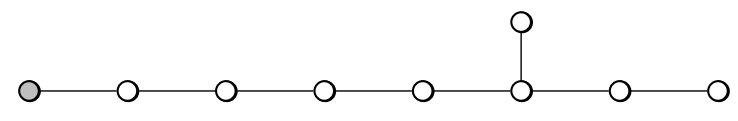

FiguRE 1. Extended Dynkin diagrams. The "extending" node $\alpha_{0}$ is depicted by a grey node $\mathrm{O}$.

contained in the $\operatorname{Aut}\left(\Delta^{+}\right)$orbit of $\alpha_{0}$. See Section 6.9 for diagrams that depict which nodes are special.

Example 6.2. If $\Delta$ is a connected Dynkin diagram of type $\mathrm{A}_{n}$, then all nodes of $\Delta$ are special. If $\Delta$ is of type $\mathrm{D}_{n}$, then all extremal nodes are special. If $\Delta$ is of type $\mathrm{E}_{8}, \mathrm{~F}_{4}$, or $\mathrm{G}_{2}$, then $\Delta$ has no special nodes.

Definition 6.3. A Deligne-Dynkin diagram over a field $Q$ is a pair $(\Delta, \mu)$, where $\Delta$ is a Dynkin diagram equipped with an action of $\Gamma_{Q}$ and $\mu$ is a subset of the special nodes of the connected components of $\Delta$ meeting each connected component in at most 1 node. (Note that $\mu$ is not required to be $\Gamma_{Q}$-stable.) The Deligne-Dynkin diagram $(\Delta, \mu)$ is called irreducible if $\pi_{0}(\Delta)$ is irreducible as a $\Gamma_{Q}$-set, and $(\Delta, \mu)$ is populated if $\mu$ meets every irreducible component of $(\Delta, \mu)$.

Remark 6.4. There is a risk of confusing terminology: a connected component of $\Delta$ or $(\Delta, \mu)$ will always mean a connected component of the Dynkin diagram $\Delta$ disregarding the $\Gamma_{Q}$-action, whereas an irreducible component $\left(\Delta^{\prime}, \mu^{\prime}\right) \subset(\Delta, \mu)$ is an irreducible $\Gamma_{Q}$-subset $\Delta^{\prime}$ of connected components of $\Delta$ with $\mu^{\prime}=\Delta^{\prime} \cap \mu$.

6.5. The reason we study Deligne-Dynkin diagrams is that we may naturally attach such a diagram to any hyperadjoint abelian motive $M$ over a field $K \subset \mathbb{C}$. These assumptions on $M$ imply that the linear algebraic group $\mathrm{G}_{\mathrm{B}}(M)$ over $\mathbb{Q}$ is an adjoint group. Let $\Delta$ be the Dynkin diagram of $\mathrm{G}_{\mathrm{B}}(M)$, and note that $\Delta$ is naturally equipped with an action of $\Gamma_{\mathbb{Q}}$. Let $\mu: \mathbb{G}_{\mathrm{m}} \rightarrow \mathrm{G}_{\mathrm{B}}(M)_{\mathbb{C}}$ be the Hodge cocharacter of the Hodge structure $\mathrm{H}_{\mathrm{B}}(M)$ (see Section 3.5). This cocharacter may be identified with a subset of nodes $\mu \subset \Delta$. Since $M$ is hyperadjoint and abelian, the computation in $[$ Del79, $\S 1.2 .5]$ shows that the nodes in $\mu$ are special and $(\Delta, \mu)$ is a populated Deligne-Dynkin diagram over $\mathbb{Q}$. We call it the Deligne-Dynkin diagram of $M$. 


\section{MTC FOR PRODUCTS OF ABELIAN VARIETIES}

6.6. We recall the definition of the opposition involution on a Dynkin diagram. Let $(R, \Phi)$ be an irreducible root system, and let $\Delta \subset \Phi$ be a choice of positive simple roots. Then $\Delta$ may be identified with the vertices of the Dynkin diagram of $(R, \Phi)$. Let $W$ be the Weyl group of $(R, \Phi)$, and let $w_{0}$ be the longest element of the Weyl group (with respect to $\Delta$ ). Then $w_{0}(\Delta)=-\Delta$, and $-w_{0}$ defines an element $\tau$ of $\operatorname{Aut}(\Delta)$ : the opposition involution. It is non-trivial if and only if $\Delta$ has type $\mathrm{A}_{k}$ with $k \neq 1, \mathrm{D}_{k}$ with $k$ odd, or $\mathrm{E}_{6}$. In these cases, $\tau$ is the unique non-trivial automorphism of $\Delta$. In particular, the opposition involution depends only on the type of $\Delta$.

For non-connected Dynkin diagrams, the opposition involution is defined componentwise.

Definition 6.7. Let $\Delta$ be a connected Dynkin diagram with opposition involution $\tau$, and let $\alpha \in \Delta$ be a special node. A node $\omega \in \Delta$ is called $\alpha$-symplectic if $\langle\alpha, \omega+\tau(\omega)\rangle=1$.

Remark 6.8. The reasoning behind this terminology is best understood in terms of Deligne's construction (Section 8, see also [Del79, § 1.3]): the $\alpha$-symplectic nodes of $\Delta$ correspond precisely to the highest weights of certain symplectic representations.

6.9. Table 1.3.9 of [Del79] lists the isomorphism classes of connected Dynkin diagrams equipped with a special node $\alpha$. In other words, these are the irreducible populated Deligne-Dynkin diagrams over an algebraically closed field $Q=\bar{Q}$. (Compared to [Del79, Table 1.3.9], we have added a diagram $\mathrm{A}_{n}$ that depicts a special case of diagram $\mathrm{A}_{p+q+1}$, for reasons of clarity. See also [Mil13, $\S 10$, in particular footnote 34$]$.)

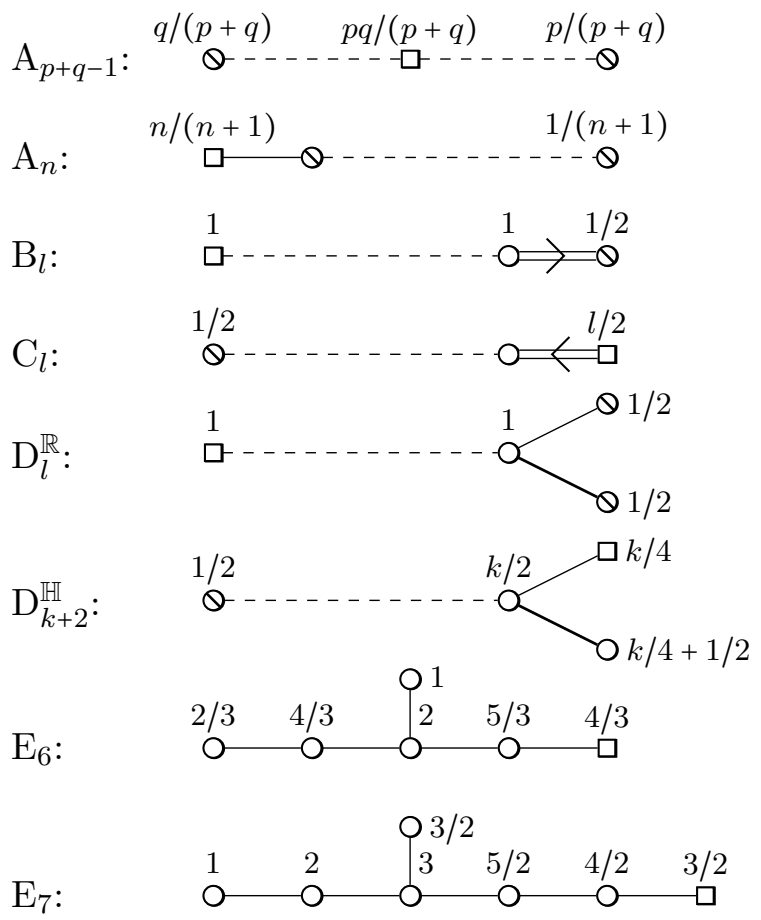

Legend (and comparison with [Del79]):

O a node (bar in [Del79])

$\square$ the special node $\alpha$ (circled node in [Del79])

$\otimes$ an $\alpha$-symplectic node (underlined node in [Del79]); see Definition 6.7

The number next to a node $\omega$ is $\langle\alpha, \omega\rangle$, where the special node $\alpha$ and the node $\omega$ are identified with the appropriate (co)characters.

In diagram $\mathrm{A}_{p+q-1}$ it is the $p$ th node that is special. If this special node is extremal, then all nodes are symplectic (diagram $\mathrm{A}_{n}$ ); otherwise, only the extremal nodes are symplectic. The diagram $\mathrm{D}_{k+2}^{\mathbb{H}}$ is required to satisfy $k+2 \geq 5$.

The diagrams of types $\mathrm{E}_{6}$ and $\mathrm{E}_{7}$ do not have symplectic nodes. The diagrams of types $\mathrm{E}_{8}, \mathrm{~F}_{4}$, and $\mathrm{G}_{2}$ do not occur: they do not have special nodes.

As explained in Example 6.2, if the Dynkin diagram has type $\mathrm{D}_{n}$, then all extremal nodes are special. This leads to two different isomorphism classes, listed in the table above, that are labelled with $\mathrm{D}_{n}^{\mathbb{R}}$ and $\mathrm{D}_{n}^{\mathbb{H}}$. The reader who has never seen these labels before should not worry about the meaning of the superscripts $\left(\_\right)^{\mathbb{R}}$ and $\left(\_\right)^{\mathbb{H}}$; the curious reader is referred to [Del79, remarque 1.3.10(ii)]. 


\section{J. Commelin}

Definition 6.10. Let $(\Delta, \mu)$ be a Deligne-Dynkin diagram over a field $Q$. The subset of $\mu$ symplectic nodes attached to $(\Delta, \mu)$ is the maximal $\Gamma_{Q}$-stable subset $S \subset \Delta$ satisfying the following condition: For every special node $\alpha \in \mu \subset \Delta$, let $\Delta_{\alpha}$ denote the connected component of $\Delta$ that contains $\alpha$. Then $S \cap \Delta_{\alpha}$ consists of only $\alpha$-symplectic nodes of $\Delta_{\alpha}$. (See also Section 6.9.)

Definition 6.11. A Deligne-Dynkin diagram $(\Delta, \mu)$ over $Q$ is symplectic if its subset of $\mu$ symplectic nodes has non-empty intersection with every irreducible component of $(\Delta, \mu)$.

Theorem 6.12. If $M$ is a hyperadjoint abelian motive over a field $K \subset \mathbb{C}$, then the DeligneDynkin diagram $(\Delta, \mu)$ associated with $M$ is symplectic.

Proof. Since $(\Delta, \mu)$ depends only on $\mathrm{H}_{\mathrm{B}}(M)$, we assume $K=\mathbb{C}$. Because $A$ is an abelian motive, there is a complex abelian variety such that $M \in\left\langle\mathrm{H}^{1}(A)\right\rangle^{\otimes}$, and thus a quotient map $\mathrm{G}_{\mathrm{B}}(A) \rightarrow$ $\mathrm{G}_{\mathrm{B}}(M)$. Hence, there is a map $\tilde{\mathrm{G}}_{\mathrm{B}}(M) \rightarrow \mathrm{G}_{\mathrm{B}}(A)^{\text {der }}$, where $\tilde{\mathrm{G}}_{\mathrm{B}}(M)$ denotes the simply connected cover of $\mathrm{G}_{\mathrm{B}}(M)$. The representation $\mathrm{G}_{\mathrm{B}}(A) \rightarrow \mathrm{GL}\left(\mathrm{H}_{\mathrm{B}}^{1}(A)\right)$ is symplectic, and thus its restriction to $\tilde{\mathrm{G}}_{\mathrm{B}}(M)$ is symplectic. In [Del79, $\S \S 1.3$ and 2.3.7], Deligne shows that this means that $(\Delta, \mu)$ is symplectic.

Example 6.13. We will now look in some detail at Deligne-Dynkin diagrams whose underlying Dynkin diagrams have connected components of type $\mathrm{D}_{4}$. Let $(\Delta, \mu)$ be such a Deligne-Dynkin diagram over some field $Q$, and assume that it is irreducible. Recall that this means that we have

- a diagram $\Delta$ consisting of a number of copies of $\mathrm{D}_{4}$,

- an action of $\Gamma_{Q}$ on $\Delta$ that is transitive on the set $\pi_{0}(\Delta)$ of connected components,

- a subset $\mu \subset \Delta$ of the extremal nodes (cf. Example 6.2) such that $\mu$ meets every connected component of $\Delta$ in at most 1 node.

Several properties of $(\Delta, \mu)$ depend on how the action of $\Gamma_{Q}$ and the subset $\mu \subset \Delta$ play together. Let $\bar{\mu}$ denote the closure of $\mu$ under the action of $\Gamma_{Q}$; then $d=\operatorname{deg}_{\pi_{0}(\Delta)}(\bar{\mu})$ takes values in $\{0,1,2,3\}$.

The cases $d=0$ and $d=3$ are somewhat degenerate. To see this, let $S \subset \Delta$ be the subset of $\mu$-symplectic nodes. If $d=0$, then $\mu=\varnothing$ and $S=\Delta$; in this case, $(\Delta, \mu)$ is not populated. On the other hand, if $(\Delta, \mu)$ is populated, then Section 6.9 shows that $S$ is contained in the set $V$ of extremal nodes of $\Delta$. In fact, we have $V=\bar{\mu} \sqcup S$ by the maximality of $S$. Hence, if $d=3$, then $S=\varnothing$ and $(\Delta, \mu)$ is not symplectic. Theorem 6.12 and Section 6.5 explain why we are not interested in these degenerate cases.

If $d=1$, then $\operatorname{deg}(S)=2$, and we say that $(\Delta, \mu)$ has type $\mathrm{D}_{4}^{\mathbb{R}}$. If $d=2$, then $\operatorname{deg}(S)=1$, and we say that $(\Delta, \mu)$ has type $D_{4}^{\mathbb{H}}$. We will not say more about these diagrams in this example, apart from mentioning that the distinction between diagrams of types $\mathrm{D}_{4}^{\mathbb{R}}$ and $\mathrm{D}_{4}^{\mathbb{H}}$ will play an important role in the proofs in the remainder of this section (notably the proof of Proposition 6.23).

6.14. The type of $(\Delta, \mu)$. Let $(\Delta, \mu)$ be an irreducible populated Deligne-Dynkin diagram over $Q$. If $(\Delta, \mu)$ is symplectic, then the type of its connected components is classical: $\mathrm{A}_{n}, \mathrm{~B}_{n}, \mathrm{C}_{n}$, or $\mathrm{D}_{n}$. Conversely, if the connected components of $\Delta$ are of type $\mathrm{A}_{n}, \mathrm{~B}_{n}$, or $\mathrm{C}_{n}$, then $(\Delta, \mu)$ is symplectic. In this case, the type of $(\Delta, \mu)$ is $\mathrm{A}_{n}, \mathrm{~B}_{n}$, or $\mathrm{C}_{n}$, respectively. The case where the connected components of $\Delta$ are of type $\mathrm{D}_{n}$ requires more attention.

Assume that the connected components of $\Delta$ are of type $\mathrm{D}_{n}$ with $n \geq 5$. For every special node $\alpha \in \mu \subset \Delta$, let $\Delta_{\alpha}$ be the connected component of $\Delta$ that contains $\alpha$. The pair $\left(\Delta_{\alpha}, \alpha\right)$ is of 


\section{MTC FOR PRODUCTS OF ABELIAN VARIETIES}

type $\mathrm{D}_{n}^{\mathbb{R}}$ or $\mathrm{D}_{n}^{\mathbb{H}}$ according to the diagrams listed in Section 6.9 . One readily verifies that $(\Delta, \mu)$ is symplectic if and only if one of the following conditions holds:

- For every special node $\alpha$, the pair $\left(\Delta_{\alpha}, \alpha\right)$ is of type $\mathrm{D}_{n}^{\mathbb{R}}$.

- For every special node $\alpha$, the pair $\left(\Delta_{\alpha}, \alpha\right)$ is of type $\mathrm{D}_{n}^{\mathbb{H}}$.

We say that $(\Delta, \mu)$ is of type $\mathrm{D}_{n}^{\mathbb{R}}$ (respectively, $\left.\mathrm{D}_{n}^{\mathbb{H}}\right)$ if the former (respectively, the latter) condition holds.

Finally, if the connected components of $\Delta$ have type $\mathrm{D}_{4}$, then the type of $(\Delta, \mu)$ was explained in Example 6.13, as follows: Let $\bar{\mu} \subset \Delta$ be the $\Gamma_{Q^{-}}$closure of $\mu$, and write $d=\operatorname{deg}_{\pi_{0}(\Delta)}(\bar{\mu})$. Recall that $d \in\{1,2,3\}$, and if $d=3$, then $(\Delta, \mu)$ is not symplectic. We say that $(\Delta, \mu)$ has type $\mathrm{D}_{4}^{\mathbb{R}}$ (respectively, $\mathrm{D}_{4}^{\mathbb{H}}$ ) if $d=1$ (respectively, $d=2$ ).

We conclude with two observations:

- The definitions of types $\mathrm{D}_{n}^{\mathbb{R}}$ and $\mathrm{D}_{n}^{\mathbb{H}}$ distinguish between the cases $n=4$ and $n \geq 5$, but these cases unify in the following way: if $(\Delta, \mu)$ is of type $\mathrm{D}_{n}^{\mathbb{R}}$ (respectively, $\mathrm{D}_{n}^{\mathbb{H}}$ ), then the subset of $\mu$-symplectic nodes of $\Delta$ has degree 2 (respectively, 1 ) over $\pi_{0}(\Delta)$.

- An irreducible symplectic populated Deligne-Dynkin diagram has one of the following types: $\mathrm{A}_{n}(n \geq 1), \mathrm{B}_{n}(n \geq 2), \mathrm{C}_{n}(n \geq 3), \mathrm{D}_{n}^{\mathbb{R}}(n \geq 4)$, or $\mathrm{D}_{n}^{\mathbb{H}}(n \geq 4)$; all these types occur.

Remark 6.15. Let $Q^{\prime} / Q$ be a field extension, and let $(\Delta, \mu)$ be a Deligne-Dynkin diagram over $Q$. By restricting the Galois action, one obtains a Deligne-Dynkin diagram $(\Delta, \mu)_{Q^{\prime}}$ over $Q^{\prime}$. We make the following observations:

(i) If $(\Delta, \mu)$ is irreducible or populated, then this need not be true for $(\Delta, \mu)_{Q^{\prime}}$. (Of course, $(\Delta, \mu)_{Q^{\prime}}$ will have irreducible components that are populated.)

(ii) Related to the preceding point: the subset of $\mu$-symplectic nodes of $(\Delta, \mu)_{Q^{\prime}}$ may be strictly larger than the subset of $\mu$-symplectic nodes of $(\Delta, \mu)$.

(iii) If $(\Delta, \mu)$ is irreducible of type $\mathrm{D}_{4}^{\mathbb{H}}$, then $(\Delta, \mu)_{Q^{\prime}}$ may have irreducible components of type $\mathrm{D}_{4}^{\mathbb{R}}$. On the other hand, if $(\Delta, \mu)$ is populated and symplectic but not of type $\mathrm{D}_{4}^{\mathbb{H}}$, then every irreducible component of $(\Delta, \mu)_{Q^{\prime}}$ that is populated must have the same type as $(\Delta, \mu)$.

Lemma 6.16. Let $(\Delta, \mu)$ be an irreducible symplectic populated Deligne-Dynkin diagram over $\mathbb{Q}$. Then there exist a prime number $\ell$ and an irreducible component of $(\Delta, \mu)_{\mathbb{Q}_{\ell}}$ that has the same type as $(\Delta, \mu)$.

Proof. By what was said in Remark 6.15(iii), this is trivial unless $(\Delta, \mu)$ has type $\mathrm{D}_{4}^{\mathbb{H}}$. In that case, let $\alpha \in \mu$ be a special node, and let $\Delta_{\alpha}$ be the connected component of $\Delta$ that contains $\alpha$. By assumption, the subset of $\mu$-symplectic nodes of $(\Delta, \mu)$ meets $\Delta_{\alpha}$ in exactly one node $s$. Label the remaining extremal node of $\Delta_{\alpha}$ with $\beta$, so that $\{\alpha, \beta, s\}$ is the set of extremal nodes of $\Delta_{\alpha}$. Note that $\bar{\mu} \cap \Delta_{\alpha}=\{\alpha, \beta\}$. Since $(\Delta, \mu)$ has type $\mathrm{D}_{4}^{\mathbb{H}}$, there exist a special node $\alpha^{\prime} \in \mu$ and an element $g \in \Gamma_{\mathbb{Q}}$ such that $g \alpha^{\prime}=\beta$. By Chebotarev's density theorem, we may assume $g \in \Gamma_{\mathbb{Q}_{\ell}}$ for some prime number $\ell$. Let $\Delta^{\prime}$ be the $\Gamma_{\mathbb{Q}_{\ell}}$-closure of $\Delta_{\alpha}$, and take $\mu^{\prime}=\Delta^{\prime} \cap \mu$. Then $\left(\Delta^{\prime}, \mu^{\prime}\right)$ has type $\mathrm{D}_{4}^{\mathbb{H}}$.

6.17. An isomorphism of Deligne-Dynkin diagrams $\phi:\left(\Delta_{1}, \mu_{1}\right) \rightarrow\left(\Delta_{2}, \mu_{2}\right)$ over a field $Q$ is a $\Gamma_{Q^{-}}$equivariant isomorphism $\phi: \Delta_{1} \rightarrow \Delta_{2}$ that maps $\mu_{1}$ onto $\mu_{2}$. We denote the set of isomorphisms from $\left(\Delta_{1}, \mu_{1}\right)$ to $\left(\Delta_{2}, \mu_{2}\right)$ by $\operatorname{Isom}\left(\left(\Delta_{1}, \mu_{1}\right),\left(\Delta_{2}, \mu_{2}\right)\right)^{\Gamma_{Q}}$. 


\section{J. Commelin}

Observe that there is a natural map $\operatorname{Isom}\left(\left(\Delta_{1}, \mu_{1}\right),\left(\Delta_{2}, \mu_{2}\right)\right)^{\Gamma_{Q}} \rightarrow \operatorname{Isom}\left(\pi_{0}\left(\Delta_{1}\right), \pi_{0}\left(\Delta_{2}\right)\right)^{\Gamma_{Q}}$. If $f \in \operatorname{Isom}\left(\pi_{0}\left(\Delta_{1}\right), \pi_{0}\left(\Delta_{2}\right)\right)^{\Gamma_{Q}}$, then we write $\operatorname{Isom}_{f}\left(\left(\Delta_{1}, \mu_{1}\right),\left(\Delta_{2}, \mu_{2}\right)\right)^{\Gamma_{Q}}$ for the set of $\phi \in$ $\operatorname{Isom}\left(\left(\Delta_{1}, \mu_{1}\right),\left(\Delta_{2}, \mu_{2}\right)\right)^{\Gamma_{Q}}$ such that $\pi_{0}(\phi)=f$.

Lemma 6.18. Let $(\Delta, \mu)$ be an irreducible symplectic populated Deligne-Dynkin diagram over $Q$. Let $\tau$ denote the opposition involution on $\Delta$. Then

$$
\text { \# Aut } \text { Aud }_{\text {id }}(\Delta, \mu)^{\Gamma_{Q}}= \begin{cases}1 & \text { if }(\Delta, \mu) \text { has type } \mathrm{A}_{1}, \mathrm{~B}_{n}, \mathrm{C}_{n}, \mathrm{D}_{n}^{\mathbb{H}}, \text { or } \mathrm{A}_{n} \text { and } \mu \text { is not fixed by } \tau, \\ 2 & \text { if }(\Delta, \mu) \text { has type } \mathrm{D}_{n}^{\mathbb{R}}, \text { or } \mathrm{A}_{n}(n \geq 2) \text { and } \mu \text { is fixed by } \tau .\end{cases}
$$

Proof. If $\Delta$ has only one connected component, then the result may be deduced from the tables in Section 6.9. If $\Delta$ has multiple components, then the result follows from the fact that a non-trivial element of $\operatorname{Aut}_{\text {id }}(\Delta, \mu)^{\Gamma_{Q}}$ must act non-trivially on every connected component of $\Delta$.

Lemma 6.19. Let $(\Delta, \mu)$ be an irreducible symplectic populated Deligne-Dynkin diagram over $\mathbb{Q}$. Then there exists an irreducible component $\left(\Delta_{\lambda}, \mu_{\lambda}\right)$ of $(\Delta, \mu)_{\mathbb{Q}_{\ell}}$ such that the natural map $\operatorname{Aut}_{\mathrm{id}}(\Delta, \mu)^{\Gamma_{\mathbb{Q}}} \rightarrow \operatorname{Aut}_{\mathrm{id}}\left(\Delta_{\lambda}, \mu_{\lambda}\right)^{\Gamma_{\mathbb{Q}}}$ is an isomorphism.

Proof. This follows immediately from Lemmas 6.16 and 6.18.

Remark 6.20. As explained at the beginning of this section, our aim is a local-global result for irreducible symplectic populated Deligne-Dynkin diagrams over $\mathbb{Q}$ (Proposition 6.23). Now recall that a quadratic extension $F$ of a number field $E$ is completely determined by the set of primes of $E$ that split in $F$. This fact inspires the following notation.

6.21. Let $(\Delta, \mu)$ be an irreducible symplectic populated Deligne-Dynkin diagram over $Q$. The action of $\Gamma_{Q}$ on $\Delta$ is determined by the action on a $\Gamma_{Q}$-closed subset $U(\Delta, \mu)$ of $\Delta$ with $\operatorname{deg}_{\pi_{0}(\Delta)}(U(\Delta, \mu)) \in\{1,2\}$. Indeed, let $S$ be the subset of $\mu$-symplectic nodes of $(\Delta, \mu)$.

- If $(\Delta, \mu)$ has type $\mathrm{A}_{n}$, we let $U(\Delta, \mu)$ be the set of extremal nodes in $\Delta$, which has degree 1 if $n=1$ and degree 2 otherwise. (Observe that this set is equal to $S$ unless all nodes in $\mu$ are extremal, cf. Section 6.9.)

- If $(\Delta, \mu)$ has type $\mathrm{B}_{n}$, we take $U(\Delta, \mu)=S$, which has degree 1 .

- If $(\Delta, \mu)$ has type $\mathrm{C}_{n}$, we take $U(\Delta, \mu)=\bar{\mu}$, which has degree 1 .

- If $(\Delta, \mu)$ has type $\mathrm{D}_{n}^{\mathbb{R}}$, we take $U(\Delta, \mu)=S$, which has degree 2 .

- If $(\Delta, \mu)$ has type $\mathrm{D}_{n}^{\mathbb{H}}$, we take $U(\Delta, \mu)=\bar{\mu}$, which has degree 1 or 2 .

6.22. Let $E$ be a number field, and let $\left(\Delta_{1}, \mu_{1}\right)$ and $\left(\Delta_{2}, \mu_{2}\right)$ be two irreducible symplectic populated Deligne-Dynkin diagrams over $\mathbb{Q}$ such that $\pi_{0}\left(\Delta_{1}\right)$ and $\pi_{0}\left(\Delta_{2}\right)$ are both isomorphic

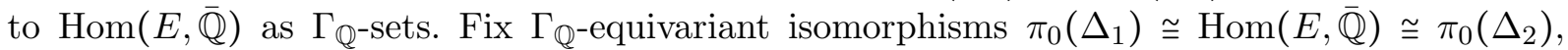
and write $f$ for the composite map $\pi_{0}\left(\Delta_{1}\right) \rightarrow \pi_{0}\left(\Delta_{2}\right)$. Suppose that there is an isomorphism $\phi \in \operatorname{Isom}_{f}\left(\left(\Delta_{1}, \mu_{1}\right),\left(\Delta_{2}, \mu_{2}\right)\right)^{\Gamma_{\mathbb{Q}}}$.

We restrict the Galois action to $\Gamma_{\mathbb{Q}_{\ell}}$ for some prime number $\ell$. The irreducible components of $\left(\Delta_{1}, \mu_{1}\right)_{\mathbb{Q}_{\ell}}$ are in a natural way indexed by the places $\lambda$ of $E$ that lie above $\ell$, so that $\left(\Delta_{1}, \mu_{1}\right)_{\mathbb{Q}_{\ell}}=\sqcup_{\lambda \mid \ell}\left(\Delta_{1}, \mu_{1}\right)_{\lambda}$. In a similar manner, the maps $f_{\ell}$ and $\phi_{\ell}$ are the disjoint union of local components $f_{\lambda}$ and $\phi_{\lambda}$. We will use this notation below.

Proposition 6.23. Let $E$ be a number field, and let $\left(\Delta_{1}, \mu_{1}\right)$ and $\left(\Delta_{2}, \mu_{2}\right)$ be two irreducible symplectic populated Deligne-Dynkin diagrams over $\mathbb{Q}$ such that $\pi_{0}\left(\Delta_{1}\right)$ and $\pi_{0}\left(\Delta_{2}\right)$ are both 


\section{MTC FOR PRODUCTS OF ABELIAN VARIETIES}

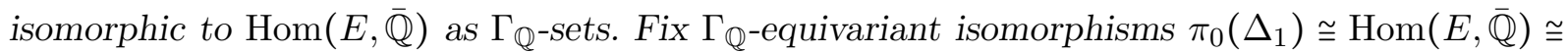
$\pi_{0}\left(\Delta_{2}\right)$, and write $f$ for the composite map $\pi_{0}\left(\Delta_{1}\right) \rightarrow \pi_{0}\left(\Delta_{2}\right)$. Suppose that for each prime number $\ell$, there is an element $\psi_{\ell} \in \operatorname{Isom}_{f}\left(\left(\Delta_{1}, \mu_{1}\right)_{\mathbb{Q}_{\ell}},\left(\Delta_{2}, \mu_{2}\right)_{\mathbb{Q}_{\ell}}\right)^{\Gamma_{\mathbb{Q}_{\ell}}}$. Then there exist an isomorphism $\phi \in \operatorname{Isom}_{f}\left(\left(\Delta_{1}, \mu_{1}\right),\left(\Delta_{2}, \mu_{2}\right)\right)^{\Gamma \mathbb{Q}}$ and a finite place $\lambda$ of $E$ such that $\phi_{\lambda}=\psi_{\lambda}$.

6.24. Unfortunately, the proof of this proposition is rather long and technical. We will first look at some easy cases that give a good idea of the strategy. After that, the remaining cases are dealt with. The proof concludes in Section 6.28.

Note that it suffices to prove the existence of $\phi$; the second claim will follow automatically from Lemma 6.19. First of all, observe that $\left(\Delta_{1}, \mu_{1}\right)$ and $\left(\Delta_{2}, \mu_{2}\right)$ have the same type, by Remark 6.15(iii) and Lemma 6.16. If this type is $\mathrm{B}_{n}$ or $\mathrm{C}_{n}$, then the topology of the diagrams and the map $f: \pi_{0}\left(\Delta_{1}\right) \rightarrow \pi_{0}\left(\Delta_{2}\right)$ determine a unique $\Gamma_{\mathbb{Q}}$-equivariant map $\phi \in \operatorname{Isom}_{f}\left(\Delta_{1}, \Delta_{2}\right)^{\Gamma}{ }_{\mathbb{Q}}$. This map $\phi$ sends $\mu_{1}$ to $\mu_{2}$ because it does so locally.

6.25. The other types require more bookkeeping, although the strategy remains the same. We begin by proving that there is an element $\phi \in \operatorname{Isom}_{f}\left(\Delta_{1}, \Delta_{2}\right)^{\Gamma_{\mathbb{Q}}}$, that may or may not map $\mu_{1}$

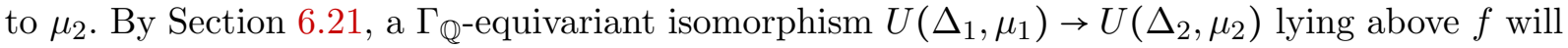
extend to a $\Gamma_{\mathbb{Q}^{-}}$-equivariant isomorphism $\Delta_{1} \rightarrow \Delta_{2}$. Since the degree of $U\left(\Delta_{i}, \mu_{i}\right)$ over $\pi_{0}\left(\Delta_{i}\right)$ is at most 2, the existence of a global isomorphism $U\left(\Delta_{1}, \mu_{1}\right) \rightarrow U\left(\Delta_{2}, \mu_{2}\right)$ will follow from Chebotarev's density theorem if we prove that these sets are locally isomorphic (cf. Remark 6.20).

- If $\left(\Delta_{1}, \mu_{1}\right)$ and $\left(\Delta_{2}, \mu_{2}\right)$ have type $\mathrm{A}_{n}$, or $\mathrm{D}_{n}^{\mathbb{R}}$ with $n \geq 5$, then the topology of the diagrams forces that $\psi_{\ell}\left(U\left(\Delta_{1}, \mu_{1}\right)\right)$ equals $U\left(\Delta_{2}, \mu_{2}\right)$ for all prime numbers $\ell$.

- Now assume that $\left(\Delta_{1}, \mu_{1}\right)$ and $\left(\Delta_{2}, \mu_{2}\right)$ have type $\mathrm{D}_{4}^{\mathbb{R}}$ or $\mathrm{D}_{4}^{\mathbb{H}}$. Let $\lambda$ be a finite place of $E$. There are two possibilities: either the extremal nodes of $\left(\Delta_{1}, \mu_{1}\right)_{\lambda}$ and $\left(\Delta_{2}, \mu_{2}\right)_{\lambda}$ form two orbits under the action of $\Gamma_{\mathbb{Q}_{\ell}}$, or the extremal nodes form three orbits. For both possibilities, it is clear that $U\left(\Delta_{1}, \mu_{1}\right)_{\lambda}$ is isomorphic to $U\left(\Delta_{2}, \mu_{2}\right)_{\lambda}$.

- Finally, assume that $\left(\Delta_{1}, \mu_{1}\right)$ and $\left(\Delta_{2}, \mu_{2}\right)$ have type $\mathrm{D}_{n}^{\mathbb{H}}$ with $n \geq 5$. Recall that the subset $U\left(\Delta_{i}, \mu_{i}\right)=\bar{\mu}_{i}$ has degree 1 or 2 . If these degrees are equal for $i=1,2$, then we are done. Now suppose that the degree of $\bar{\mu}_{1}$ is 2 . Let $\{\alpha, \beta\} \subset \bar{\mu}_{1}$ be two nodes that lie in the same connected component of $\Delta_{1}$. Without loss of generality, we may assume $\alpha \in \mu_{1}$. By Chebotarev's density theorem, there exist a prime number $\ell$ and a $g \in \Gamma_{\mathbb{Q}_{\ell}}$ such that $g \alpha=\beta$. By assumption, we have $\psi_{\ell}(\alpha) \in \mu_{2}$ and $\psi_{\ell}(\beta)=g \psi_{\ell}(\alpha) \in \bar{\mu}_{2}$. We conclude that $\bar{\mu}_{1}$ and $\bar{\mu}_{2}$ have the same degree.

We have now proven that there exists a $\Gamma_{\mathbb{Q}^{-}}$equivariant isomorphism $\phi: \Delta_{1} \rightarrow \Delta_{2}$ lying over $f$. It remains to prove that we can choose $\phi$ in such a way that it maps $\mu_{1}$ to $\mu_{2}$.

6.26. At the beginning of this proof, we already dealt with the cases where $\left(\Delta_{1}, \mu_{1}\right)$ and $\left(\Delta_{2}, \mu_{2}\right)$ have type $\mathrm{B}_{n}$ or $\mathrm{C}_{n}$. We continue with some other easy cases, where the topology of the diagrams forces $\phi\left(\mu_{1}\right)=\mu_{2}$. This happens

- if $\left(\Delta_{1}, \mu_{1}\right)$ and $\left(\Delta_{2}, \mu_{2}\right)$ are of type $\mathrm{A}_{1}$, or $\mathrm{D}_{n}^{\mathbb{R}}$ with $n \geq 5$;

- if the type is $\mathrm{D}_{4}^{\mathbb{R}}$, since $\phi$ maps $U\left(\Delta_{1}, \mu_{1}\right)=S_{1}$ to $U\left(\Delta_{2}, \mu_{2}\right)=S_{2}$ by construction; and

- if the type is $\mathrm{A}_{n}$ with $n \geq 2$, and $\mu_{1}$ (and thus $\mu_{2}$ ) is fixed by the opposition involution.

The remaining cases require a bit more work: so far we have not needed to change our choice 


\section{J. Commelin}

of $\phi$, but with the remaining cases, this might be necessary.

6.27. First consider the case $D_{4}^{\mathbb{H}}$. Recall that $\bar{\mu}_{i}$ has degree 2 . Thus there is a unique non-trivial $\Gamma_{\mathbb{Q}^{-}}$equivariant involution $\tau_{i} \in \operatorname{Aut}_{\mathrm{id}}\left(\Delta_{i}\right)$ such that $\tau_{i}\left(\bar{\mu}_{i}\right)=\bar{\mu}_{i}$. Observe that $\phi \circ \tau_{1}=\tau_{2} \circ \phi$. Let $\alpha \in \mu_{1}$ be a special node. We may assume $\phi(\alpha) \in \mu_{2}$ by replacing $\phi$ with $\tau_{2} \circ \phi$ if necessary.

We claim that $\phi\left(\mu_{1}\right)=\mu_{2}$. Indeed, let $\alpha^{\prime} \in \mu_{1}$ be a special node. Recall from Example 6.13 that $\operatorname{deg}\left(\bar{\mu}_{i}\right)=2$. Now there are two cases: either $\alpha$ and $\alpha^{\prime}$ are in the same $\Gamma_{\mathbb{Q}}$-orbit, or they are in different orbits. Assume that $\alpha$ and $\alpha^{\prime}$ are in the same orbit. The proof of this case contains the essential idea that will also be applied in all the remaining cases: There exists a $g \in \Gamma_{\mathbb{Q}}$ such that $g \alpha=\alpha^{\prime}$. By Chebotarev's density theorem, we may assume $g \in \Gamma_{\mathbb{Q}_{\ell}}$ for some prime number $\ell$. Since $\phi(\alpha) \in \mu_{2}$, we have $\phi(\alpha)=\psi_{\ell}(\alpha)$, and we compute

$$
\phi\left(\alpha^{\prime}\right)=\phi(g \alpha)=g \phi(\alpha)=g \psi_{\ell}(\alpha)=\psi_{\ell}(g \alpha)=\psi_{\ell}\left(\alpha^{\prime}\right) \in \mu_{2} .
$$

Now suppose that $\alpha$ and $\alpha^{\prime}$ are in different orbits. Let $\Delta_{\alpha}$ and $\Delta_{\alpha^{\prime}}$ be the connected components of $\Delta$ that contain $\alpha$ and $\alpha^{\prime}$, respectively. Let $s$ and $s^{\prime}$ be the $\mu$-symplectic nodes in $\Delta_{\alpha}$ and $\Delta_{\alpha^{\prime}}$, respectively. Finally, let $\beta \in \Delta_{\alpha}$ and $\beta^{\prime} \in \Delta_{\alpha^{\prime}}$ be such that $\{\alpha, \beta, s\}$ is the set of extremal nodes of $\Delta_{\alpha}$ and $\left\{\alpha^{\prime}, \beta^{\prime}, s^{\prime}\right\}$ is the set of extremal nodes of $\Delta_{\alpha^{\prime}}$. There exists a $g \in \Gamma_{\mathbb{Q}}$ such that $g \alpha=\beta^{\prime}$. By Chebotarev's density theorem, we may assume $g \in \Gamma_{\mathbb{Q}_{\ell}}$ for some prime number $\ell$. Let $\lambda \mid \ell$ be the finite place of $E$ that corresponds to the $\Gamma_{\mathbb{Q}_{\ell}}$-closure of $\Delta_{\alpha}$.

By construction, $\phi$ maps $U\left(\Delta_{1}, \mu_{1}\right)=\bar{\mu}_{1}$ to $U\left(\Delta_{2}, \mu_{2}\right)=\bar{\mu}_{2}$. On the other hand, we have $\psi_{\lambda}\left(\bar{\mu}_{1, \lambda}\right)=\bar{\mu}_{2, \lambda}$ by assumption; thus $\phi(s)=\phi_{\lambda}(s)$. Since $\phi(\alpha) \in \mu_{2}$, we have $\phi(\alpha)=\psi_{\lambda}(\alpha)$, and therefore $\phi(\beta)=\psi_{\lambda}(\beta)$. We compute $\phi\left(\alpha^{\prime}\right)=\phi(g \beta)=g \phi(\beta)=g \psi_{\lambda}(\beta)=\psi_{\lambda}(g \beta)=\psi_{\lambda}\left(\alpha^{\prime}\right) \in \mu_{2}$. This finishes the proof in the case $\mathrm{D}_{4}^{\mathbb{H}}$.

6.28. Finally, suppose that the type is $\mathrm{D}_{n}^{\mathbb{H}}$ with $n \geq 5$, or the type is $\mathrm{A}_{n}$ and $\mu_{i}$ is not fixed by the opposition involution. The proof is similar to that in the case $\mathrm{D}_{4}^{\mathbb{H}}$. There is a unique non-trivial $\Gamma_{\mathbb{Q}^{-}}$equivariant involution $\tau_{i} \in \operatorname{Aut}_{i d}\left(\Delta_{i}\right)$. Once again, we have $\phi \circ \tau_{1}=\tau_{2} \circ \phi$. Let $\alpha \in \mu_{1}$ be a special node that is not fixed under $\tau_{1}$. We may assume $\phi(\alpha) \in \mu_{2}$ by replacing $\phi$ with $\tau_{2} \circ \phi$ if necessary.

As before, we claim that $\phi\left(\mu_{1}\right)=\mu_{2}$. Indeed, suppose that $\alpha^{\prime} \in \mu_{1}$ is a special node, and let $\Delta_{\alpha^{\prime}}$ be the component of $\Delta_{1}$ containing $\alpha^{\prime}$. There exists a $g \in \Gamma_{\mathbb{Q}}$ such that $g \alpha \in \Delta_{\alpha^{\prime}}$. By Chebotarev's density theorem, we may assume $g \in \Gamma_{\mathbb{Q}_{\ell}}$ for some prime number $\ell$. Now we argue as follows: Let $\left(\Delta_{1}, \mu_{1}\right)_{\lambda}$ (respectively, $\left.\left(\Delta_{2}, \mu_{2}\right)_{\lambda}\right)$ be the irreducible component of $\left(\Delta_{1}, \mu_{1}\right)_{\mathbb{Q}_{\ell}}$ (respectively, $\left.\left(\Delta_{2}, \mu_{2}\right)_{\mathbb{Q}_{\ell}}\right)$ that contains $\alpha$ (respectively, $\phi(\alpha)$ ). Since $\phi(\alpha) \in \mu_{2}$, we have $\phi(\alpha)=\psi_{\ell}(\alpha)$ and therefore $\phi_{\lambda}=\psi_{\lambda}$. This implies $\phi\left(\alpha^{\prime}\right) \in \mu_{2}$ by a computation similar to the one above. This completes the proof of Proposition 6.23.

Remark 6.29. In Proposition 6.23, the hypothesis that $\psi_{\ell}$ exists for each prime number $\ell$ may be weakened to the condition that $\psi_{\ell}$ exists for a set of prime numbers with Dirichlet density 1. This weaker condition is sufficient to apply Chebotarev's density theorem in Section 6.25.

\section{Deligne-Dynkin diagrams and $p$-adic Hodge theory}

Let $M$ be a hyperadjoint abelian motive over a finitely generated field $K \subset \mathbb{C}$, and let $(\Delta, \mu)$ be the Deligne-Dynkin diagram of $M$. In this short section, we apply $p$-adic Hodge theory to determine the subset $\mu \subset \Delta$ from the $p$-adic realisation $\mathrm{H}_{p}(M)$. 


\section{MTC FOR PRODUCTS OF ABELIAN VARIETIES}

Theorem 7.1. Let $M$ be a motive over a $p$-adic field $K_{v}$. Let $C_{v}$ be the completion of an algebraic closure of $K_{v}$, and for $i \in \mathbb{Z}$, write $C_{v}(i)$ for $C_{v} \otimes_{\mathbb{Q}_{p}} \mathbb{Q}_{p}(i)$ with the diagonal action of $\Gamma_{K_{v}}$. Then there is a functorial isomorphism of $C_{v}$-vector spaces

$$
\mathrm{H}_{p}(M) \otimes_{\mathbb{Q}_{p}} C_{v} \cong \bigoplus_{i \in \mathbb{Z}} \operatorname{gr}^{i} \mathrm{H}_{\mathrm{dR}}(M) \otimes_{K_{v}} C_{v}(-i)
$$

that is compatible with the action of $\Gamma_{K_{v}}$.

Proof. If $M=\mathrm{H}^{n}(X)$ for some smooth projective variety $X$ over $K_{v}$, then this result is [Fal88, Theorem 4.1]. Since the cited theorem shows that the comparison isomorphism is an isomorphism of Weil cohomology theories, one deduces that the isomorphism is compatible with motivated cycles. This implies that the result is true for a general motive $M$.

Corollary 7.2. Let $\mathrm{B}_{\mathrm{HT}, v}$ denote the graded ring $\oplus_{i \in \mathbb{Z}} C_{v}(i)$, and let $\mathrm{F}_{\mathrm{HT}, v}$ be the field of fractions of $\mathrm{B}_{\mathrm{HT}, v}$. Then there is a functorial isomorphism of graded $\mathrm{F}_{\mathrm{HT}, v}$-vector spaces

$$
\left(\mathrm{H}_{p}(M) \otimes_{\mathbb{Q}_{p}} \mathrm{~B}_{\mathrm{HT}, v}\right) \otimes_{\mathrm{B}_{\mathrm{HT}, v}} \mathrm{~F}_{\mathrm{HT}, v} \cong \operatorname{gr~}_{\mathrm{dR}}(M) \otimes_{K_{v}} \mathrm{~F}_{\mathrm{HT}, v}
$$

that is compatible with the action of $\Gamma_{K_{v}}$. (The grading on the left-hand-side comes from the grading on $\mathrm{B}_{\mathrm{HT}, v}$.)

7.3. Let $M$ be a motive over a finitely generated field $K \subset \mathbb{C}$, and choose an embedding of $K$ into a $p$-adic field $K_{v}$. Let $C_{v}, C_{v}(i), \mathrm{B}_{\mathrm{HT}, v}$, and $\mathrm{F}_{\mathrm{HT}, v}$ be as above. The choices $K \subset K_{v} \subset C_{v}$ determine a grading on $\mathrm{H}_{p}(M) \otimes_{\mathbb{Q}_{p}} \mathrm{~F}_{\mathrm{HT}, v}$. This grading induces a cocharacter $\mu_{\mathrm{HT}}$ of $\mathrm{G}_{p}(M) \otimes_{\mathbb{Q}_{p}}$ $\mathrm{F}_{\mathrm{HT}, v} \subset \mathrm{G}(M) \otimes_{\mathbb{Q}} \mathrm{F}_{\mathrm{HT}, v}$ that is called the Hodge-Tate cocharacter. If $M$ is abelian, then we may view $\mu_{\mathrm{HT}}$ as a cocharacter of $\mathrm{G}_{\mathrm{B}}(M) \otimes_{\mathbb{Q}} \mathrm{F}_{\mathrm{HT}, v}$ since $\mathrm{G}_{p}^{\circ}(M) \subset \mathrm{G}_{\mathrm{B}}(M) \otimes_{\mathbb{Q}} \mathbb{Q}_{p}=\mathrm{G}(M)^{\circ} \otimes_{\mathbb{Q}} \mathbb{Q}_{p}$ by Theorem 4.13.

Lemma 7.4. Let $M$ be a hyperadjoint abelian motive over a finitely generated field $K \subset \mathbb{C}$, and choose an embedding of $K$ into a p-adic field $K_{v}$. Let $C_{v}, C_{v}(i), \mathrm{B}_{\mathrm{HT}, v}$, and $\mathrm{F}_{\mathrm{HT}, v}$ be as above. Then the $\mathrm{G}_{\mathrm{B}}(M)$-conjugacy class of $\mu_{\mathrm{HT}} \in \mathrm{X}_{*}\left(\mathrm{G}_{\mathrm{B}}(M) \otimes_{\mathbb{Q}} \mathrm{F}_{\mathrm{HT}, v}\right)$ and the $\mathrm{G}_{\mathrm{B}}(M)$-conjugacy class of the Hodge cocharacter $\mu \in \mathrm{X}_{*}\left(\mathrm{G}_{\mathrm{B}}(M) \otimes_{\mathbb{Q}} \mathbb{C}\right)$ both correspond to the same $\mathrm{G}_{\mathrm{B}}(M)$-conjugacy class of cocharacters in $\mathrm{X}_{*}\left(\mathrm{G}_{\mathrm{B}}(M) \otimes_{\mathbb{Q}} \overline{\mathbb{Q}}\right)$. In particular, if $(\Delta, \mu)$ is the Deligne-Dynkin diagram of $M$, then $\mu_{\mathrm{HT}}$ determines the subset $\mu \subset \Delta$.

Proof. By the Tannakian formalism, the two cocharacters $\mu_{\mathrm{HT}}$ and $\mu$ correspond to two tensor functors, namely the realisation functors

$$
\begin{aligned}
& \mathrm{H}_{p} \otimes \mathrm{F}_{\mathrm{HT}, v}:\langle M\rangle^{\otimes} \rightarrow \operatorname{grVect}_{\mathrm{F}_{\mathrm{HT}, v}}, \\
& N \mapsto \mathrm{H}_{p}(N) \otimes_{\mathbb{Q}_{p}} \mathrm{~F}_{\mathrm{HT}, v}, \\
& \mathrm{H}_{\mathrm{B}} \otimes \mathbb{C}:\langle M\rangle^{\otimes} \rightarrow \operatorname{grVect}_{\mathbb{C}}, \\
& N \mapsto \mathrm{H}_{\mathrm{B}}(N) \otimes_{\mathbb{Q}} \mathbb{C} .
\end{aligned}
$$

There is also another tensor functor

$$
\begin{aligned}
\operatorname{grH}_{\mathrm{dR}}:\langle M\rangle^{\otimes} & \rightarrow \operatorname{grVect}_{K}, \\
N & \mapsto \operatorname{grH}_{\mathrm{dR}}(N)
\end{aligned}
$$

that maps a motive to the associated graded vector space of its algebraic de Rham realisation. Write $U_{K}$ for the forgetful functor grVect $_{K} \rightarrow \operatorname{Vect}_{K}$. If $\mathcal{G}(M) / K$ denotes the algebraic group $\underline{\mathrm{Aut}}^{\otimes}\left(U_{K} \circ \mathrm{grH}_{\mathrm{dR}}\right)$ that corresponds to $\langle M\rangle^{\otimes}$ via the fibre functor $U_{K} \circ \mathrm{gr} \mathrm{H}_{\mathrm{dR}}$, then gr $\mathrm{H}_{\mathrm{dR}}$ corresponds to a cocharacter $\mu_{\mathrm{dR}} \in \mathrm{X}_{*}(\mathcal{G}(M))$.

The comparison theorem between the Betti realisation and the algebraic de Rham realisation gives a natural transformation $\mathrm{H}_{\mathrm{B}} \otimes \mathbb{C} \rightarrow \operatorname{gr} \mathrm{H}_{\mathrm{dR}} \otimes \mathbb{C}$. This yields an injective homomorphism 


\section{J. Commelin}

of complex algebraic groups $\mathrm{G}_{\mathrm{B}}(M) \otimes_{\mathbb{Q}} \mathbb{C} \hookrightarrow \mathcal{G}(M) \otimes_{K} \mathbb{C}$ that maps $\mu$ to $\mu_{\mathrm{dR}}$. On the other hand, $p$-adic Hodge theory compares the $p$-adic étale realisation with the algebraic de Rham realisation and gives a natural transformation $\mathrm{H}_{p} \otimes \mathrm{F}_{\mathrm{HT}, v} \rightarrow \operatorname{gr} \mathrm{H}_{\mathrm{dR}} \otimes \mathrm{F}_{\mathrm{HT}, v}$. This yields an injection $\mathrm{G}_{\mathrm{B}}(M) \otimes_{\mathbb{Q}} \mathrm{F}_{\mathrm{HT}, v} \hookrightarrow \mathcal{G}(M) \otimes_{K} \mathrm{~F}_{\mathrm{HT}, v}$ that maps $\mu_{\mathrm{HT}}$ to $\mu_{\mathrm{dR}}$.

The proof concludes by an application of the following remark: Let $\bar{Q} \subset \bar{Q}^{\prime}$ be an extension of algebraically closed fields of characteristic 0 , and let $G$ be an algebraic group over $\bar{Q}$. Then the natural map $\mathrm{X}_{*}(G) \rightarrow \mathrm{X}_{*}\left(G_{\bar{Q}^{\prime}}\right)$ obtained by base change induces a bijection between the conjugacy classes of cocharacters of $G$ and the conjugacy classes of cocharacters of $G_{\bar{Q}^{\prime}}$. Indeed, let $T \subset B \subset G$ be a choice of a maximal torus $T$ and a Borel $\operatorname{subgroup~} B$, and let Fund $(T, B) \subset$ $\mathrm{X}_{*}(T)$ denote the fundamental chamber. Every conjugacy class of cocharacters of $G$ has a unique representative in $\operatorname{Fund}(T, B)$, and so we find a chain of isomorphisms

$$
\mathrm{X}_{*}(G) / G \cong \operatorname{Fund}(T, B) \cong \operatorname{Fund}\left(T_{\bar{Q}^{\prime}}, B_{\bar{Q}^{\prime}}\right) \cong \mathrm{X}_{*}\left(G_{\bar{Q}^{\prime}}\right) / G_{\bar{Q}^{\prime}}
$$

This finishes the proof of the lemma.

\section{Deligne's construction}

Let $M$ be an irreducible hyperadjoint abelian motive over $\mathbb{C}$. Proposition 2.3 .10 of [Del79] provides a recipe to construct a complex abelian variety $A$ (up to isogeny) such that $M \cong \mathrm{H}^{1}(A)^{\text {ha }}$. Deligne uses the language of Shimura data and Hodge theory. We recall this construction using the terminology of abelian motives and Deligne-Dynkin diagrams.

8.1. Preparations. Let $M$ be an irreducible hyperadjoint abelian motive over $\mathbb{C}$, and let $(\Delta, \mu)$ be the Deligne-Dynkin diagram associated with $M$. Since $M$ is hyperadjoint, the group $\mathrm{G}(M)$ is adjoint, and because $M$ is irreducible, it is of the form $\operatorname{Res}_{E / \mathbb{Q}} \mathcal{G}$, where $E$ is a number field and $\mathcal{G}$ is an absolutely simple adjoint group over $E$. This number field $E$ is a totally real field (see the discussion in $[\operatorname{Del} 79, \S 2.3 .4(\mathrm{a})]$ ). We have $E=\operatorname{End}(M)$, once again because $M$ is hyperadjoint - and therefore corresponds to the adjoint representation of $\mathrm{G}(M)$ under the Tannakian formalism. Note that $\operatorname{Hom}(E, \overline{\mathbb{Q}}) \cong \pi_{0}(\Delta)$ as $\Gamma_{\mathbb{Q}^{-}}$-sets. We also recall that $(\Delta, \mu)$ is symplectic (Theorem 6.12). Let $S$ be the subset of $\mu$-symplectic nodes of $(\Delta, \mu)$.

Write $G$ for the $\mathbb{Q}$-simple adjoint group $\mathrm{G}_{\mathrm{B}}(M)$, and let $\tilde{G}$ be the simply connected cover of $G$. For $s \in S$, let $V(s)$ be the representation of $\tilde{G}_{\mathbb{C}}$ whose highest weight corresponds to $s$. Since $S$ is closed under the action of $\Gamma_{\mathbb{Q}}$, there exists a representation $V$ of $\tilde{G}$ over $\mathbb{Q}$ such that $V_{\mathbb{C}} \cong \bigoplus_{s \in S} V(s)^{\oplus n}$ for suitable $n$.

8.2. Choices. Now we fix three choices:

(1) Choose a totally imaginary quadratic extension $F / E$.

(2) Choose a partial CM type $\Phi$ for $F$ relative to $(\Delta, \mu)$ : a subset $\Phi \subset \operatorname{Hom}(F, \mathbb{C})=\operatorname{Hom}(F, \overline{\mathbb{Q}})$ that maps 1-to-1 onto the complement of the image of $\mu$ in $\operatorname{Hom}(E, \overline{\mathbb{Q}})=\pi_{0}(\Delta)$.

(3) Choose a representation $V$ of $\tilde{G}$ as above.

8.3. The Hodge cocharacter $h: \mathbb{S} \rightarrow G_{\mathbb{R}}$ lifts to a map $\tilde{h}: \tilde{\mathbb{S}} \rightarrow \tilde{G}_{\mathbb{R}}$, endowing $V$ with a fractional Hodge structure. The Hodge decomposition of $V$ may be read off from the diagrams in Section 6.9: If $s \in S$ lies in a component of $\Delta$ that does not meet $\mu$, then the type of $V(s)$ is $\{(0,0)\}$. If $s$ lies in a component of $\Delta$ that contains a special node $\alpha \in \mu$, then the type of $V(s)$ is $\{(r,-r),(r-1,1-r)\}$, 
where $r=\langle s, \alpha\rangle$ is the number that is written next to the node $s$ in the appropriate diagram in Section 6.9.

8.4. Let $F_{S}$ denote the étale $E$-algebra such that $\operatorname{Hom}\left(F_{S}, \overline{\mathbb{Q}}\right) \cong S$ as $\Gamma_{\mathbb{Q}^{-}}$-sets. Observe that the fractional Hodge structure $V$ is canonically an $F_{S}$-module: the algebra $F_{S}$ acts on $V(s)$ via the embedding $F_{S} \hookrightarrow \overline{\mathbb{Q}} \subset \mathbb{C}$ that corresponds to $s \in S$.

We endow $F_{S}$ with a fractional pre-Hodge structure: the component $\mathbb{C}^{\{s\}}$ of $F_{S} \otimes_{\mathbb{Q}} \mathbb{C} \cong \mathbb{C}^{S}$ is placed in bi-degree $(0,0)$ if $s$ lies in a component of $\Delta$ that does not meet $\mu$, and $\mathbb{C}^{\{s\}}$ is placed in bi-degree $(1-r, r)$ if $s$ lies in a component that does meet $\mu$, where $r$ is the rational number from the preceding paragraph.

In a similar fashion, we endow the $\mathrm{CM}$ field $F$ with a fractional pre-Hodge structure: the component $\mathbb{C}^{\phi}$ of $F \otimes \mathbb{C} \cong \mathbb{C}^{\operatorname{Hom}(F, \mathbb{C})}$ is placed in bi-degree

$$
\begin{cases}(1,0) & \text { if } \phi \in \Phi \\ (0,1) & \text { if } \bar{\phi} \in \Phi \\ (0,0) & \text { otherwise. }\end{cases}
$$

8.5. Write $W_{F}$ for $F \otimes_{E} F_{S}$, and observe that $F \otimes_{E} F_{S}$ is a fractional Hodge structure of weight 1 . Note that $W_{F}$ is of CM type since both $F$ and $F_{S}$ are of CM type. Put $V^{\prime}=W_{F} \otimes_{F_{S}} V$. A computation shows that $V^{\prime}$ is a Hodge structure of type $\{(1,0),(0,1)\}$. It turns out that $V^{\prime}$ is a polarisable Hodge structure (see [Del79, corollaire 2.3.3] for details). Thus there is a complex abelian variety $A$ (well-defined up to isogeny) such that $\mathrm{H}_{\mathrm{B}}^{1}(A) \cong V^{\prime}$. Since $W_{F}$ is of CM type, we find that $\mathrm{G}_{\mathrm{B}}\left(V^{\prime}\right)^{\text {ad }}=G$, and therefore $\mathrm{H}^{1}(A)^{\text {ha }}=M$.

8.6. Write $T$ for the torus $\mathrm{G}_{\mathrm{B}}(A)^{\mathrm{ab}}=\mathrm{G}_{\mathrm{B}}\left(V^{\prime}\right)^{\mathrm{ab}}$. Choose a faithful representation $N$ of $T$; by the Tannakian formalism and Theorem 4.13, we may view $N$ as an abelian CM motive over $\mathbb{C}$.

Since $\mathrm{G}_{\mathrm{B}}\left(W_{F}\right)$ is a torus and $\tilde{G}$ is almost simple, the representation

$$
\mathrm{G}_{\mathrm{B}}\left(W_{F}\right) \times \tilde{G} \rightarrow \mathrm{GL}\left(V^{\prime}\right)=\mathrm{GL}\left(W_{F} \otimes_{F_{S}} V\right)
$$

has finite kernel. In other words, the natural map $\mathrm{G}_{\mathrm{B}}\left(W_{F}\right) \times \tilde{G} \rightarrow \mathrm{G}_{\mathrm{B}}(A)$ is an isogeny, and so is the natural map $\mathrm{G}_{\mathrm{B}}\left(W_{F}\right) \rightarrow T$. We will need this fact in Section 9.8.

\section{The main proposition}

In this section, we prove the main technical result of this paper. Its proof uses results of the preceding three sections.

Proposition 9.1. Let $M_{1}$ and $M_{2}$ be two geometrically irreducible hyperadjoint abelian motives over a finitely generated field $K \subset \mathbb{C}$. Assume that $\operatorname{MTC}\left(M_{1}\right)$ and $\operatorname{MTC}\left(M_{2}\right)$ are true, and assume that $\mathrm{G}_{\ell}\left(M_{1} \oplus M_{2}\right)$ is connected for all prime numbers $\ell$. If there exists a prime number $\ell$ such that $\mathrm{G}_{\ell}\left(M_{1} \oplus M_{2}\right) \mp \mathrm{G}_{\ell}\left(M_{1}\right) \times \mathrm{G}_{\ell}\left(M_{2}\right)$, then $\mathrm{H}_{\mathrm{B}}\left(M_{1}\right) \cong \mathrm{H}_{\mathrm{B}}\left(M_{2}\right)$ as Hodge structures.

9.2. The proof of this proposition will take the remainder of this section. Roughly speaking, the strategy is as follows:

(1) First we prove that $\operatorname{End}\left(M_{1}\right)=\operatorname{End}\left(M_{2}\right)$.

(2) Next we show that $\mathrm{H}_{\ell}\left(M_{1}\right) \cong \mathrm{H}_{\ell}\left(M_{2}\right)$ for all primes $\ell$. 


\section{J. Commelin}

(3) We use this (and Proposition 6.23) to show that $M_{1}$ and $M_{2}$ have isomorphic DeligneDynkin diagrams over $\mathbb{Q}$.

(4) After that we run Deligne's construction (Section 8) on the motives $M_{i}$; this leaves us with two complex abelian varieties $A_{1}$ and $A_{2}$ such that $M_{i, \mathbb{C}}=\mathrm{H}^{1}\left(A_{i}\right)^{\text {ha }}$.

(5) We replace $K$ with a finitely generated extension such that $A_{1}$ and $A_{2}$ are defined over $K$.

(6) By carefully tracing the $\ell$-adic counterpart of the construction, we show that $A_{1}$ and $A_{2}$ have isomorphic $\ell$-adic Tate modules.

(7) Finally, we apply Faltings's results to deduce that $A_{1}$ and $A_{2}$ are isogenous abelian varieties, which implies $\mathrm{H}_{\mathrm{B}}\left(M_{1}\right) \cong \mathrm{H}_{\mathrm{B}}\left(M_{2}\right)$.

Sadly, however, this strategy is slightly too optimistic. It is not possible to work with the entire $\ell$-adic Galois representations: we will have to focus our attention on a suitable summand. This makes the proof quite technical.

9.3. We first make some observations about $M_{1}$ and $M_{2}$. For $i \in\{1,2\}$, write $E_{i}$ for $\operatorname{End}\left(M_{i}\right)$, and write $\Lambda_{i}$ for the set of finite places of $E_{i}$. Observe that $\mathrm{H}_{\Lambda_{i}}\left(M_{i}\right)$ is a quasi-compatible system of representations, by Theorem 5.13 .

For a prime number $\ell$, let $\Lambda_{i, \ell}$ denote the set of places $\lambda \in \Lambda_{i}$ that lie above $\ell$. Recall that $\mathrm{G}_{\mathrm{B}}(M)=\operatorname{Res}_{E_{i} / \mathbb{Q}}\left(G_{i}\right)$ for some absolutely simple adjoint group $G_{i}$ over $E_{i}$. Since $\operatorname{MTC}\left(M_{i}\right)$ holds and Weil restriction of scalars commutes with base change, we find that

$$
\mathrm{G}_{\ell}^{\circ}\left(M_{i}\right)=\mathrm{G}_{\mathrm{B}}(M) \otimes_{\mathbb{Q}} \mathbb{Q}_{\ell}=\prod_{\lambda \in \Lambda_{i, \ell}} \operatorname{Res}_{E_{i, \lambda} / \mathbb{Q}_{\ell}}\left(G_{i} \otimes_{E_{i}} E_{i, \lambda}\right) .
$$

In particular, we have $\mathrm{G}_{\ell}\left(\mathrm{H}_{\lambda}\left(M_{i}\right)\right)=\operatorname{Res}_{E_{i, \lambda} / \mathbb{Q}_{\ell}}\left(G_{i} \otimes_{E, i} E_{i, \lambda}\right)$ for every $\lambda \in \Lambda_{i, \ell}$. If we write $\mathrm{G}_{\lambda}\left(M_{i}\right)$ for $\mathrm{G}_{\lambda}\left(\mathrm{H}_{\lambda}\left(M_{i}\right)\right)$, then the above computation implies that $\mathrm{G}_{\lambda}\left(M_{i}\right)$ is equal to $G_{i} \otimes_{E_{i}} E_{i, \lambda}$, an absolutely simple adjoint group over $E_{i, \lambda}$.

Let $\ell$ be a prime number such that $\mathrm{G}_{\ell}\left(M_{1} \oplus M_{2}\right) \mp \mathrm{G}_{\ell}\left(M_{1}\right) \times \mathrm{G}_{\ell}\left(M_{2}\right)$. By Goursat's lemma (see Remark 1.3(ii)), this implies that there are places $\lambda_{1} \in \Lambda_{1, \ell}$ and $\lambda_{2} \in \Lambda_{2, \ell}$ such that the projection of $\mathrm{G}_{\ell}\left(M_{1} \oplus M_{2}\right)$ in $\operatorname{Res}_{E_{1, \lambda_{1}} / \mathbb{Q}_{\ell}} \mathrm{G}_{\lambda_{1}}\left(M_{1}\right) \times \operatorname{Res}_{E_{2, \lambda_{2}} / \mathbb{Q}_{\ell}} \mathrm{G}_{\lambda_{2}}\left(M_{2}\right)$ is the graph of an isomorphism of algebraic groups over $\mathbb{Q}_{\ell}$. The derivative of this isomorphism at the identity element is a $\mathbb{Q}_{\ell}$-linear isomorphism $\psi: \mathrm{H}_{\lambda_{1}}\left(M_{1}\right) \rightarrow \mathrm{H}_{\lambda_{2}}\left(M_{2}\right)$ of Galois representations of $K$. (Recall that $\mathrm{H}_{\lambda_{i}}\left(M_{i}\right)$ is the adjoint representation $\operatorname{Lie}\left(\operatorname{Res}_{E_{i, \lambda_{i}} / \mathbb{Q}_{\ell}} \mathrm{G}_{\lambda_{i}}\left(M_{i}\right)\right)$.)

Note that $\psi$ induces an isomorphism $f: \operatorname{End}_{\mathbb{Q}_{\ell}}\left(\mathrm{H}_{\lambda_{1}}\left(M_{1}\right)\right)^{\Gamma_{K}} \rightarrow \operatorname{End}_{\mathbb{Q}_{\ell}}\left(\mathrm{H}_{\lambda_{2}}\left(M_{2}\right)\right)^{\Gamma_{K}}$. Since these endomorphism algebras are commutative, the isomorphism $f$ does not depend on $\psi$. By Proposition 5.15, we recover $\lambda_{i}: E_{i} \hookrightarrow E_{i, \lambda_{i}}=\operatorname{End}_{\mathbb{Q}_{\ell}}\left(\mathrm{H}_{\lambda_{i}}\left(M_{i}\right)\right)^{\Gamma_{K}}$ as the subfield generated by coefficients of characteristic polynomials of Frobenius elements acting $E_{i, \lambda_{i}}$-linearly on $\mathrm{H}_{\lambda_{i}}\left(M_{i}\right)$. Because $\psi$ commutes with the action of these Frobenius elements, the map $f$ restricts to a canonical isomorphism $f: E_{1} \rightarrow E_{2}$ that identifies $\lambda_{1}$ with $\lambda_{2}$. Write $E$ for $E_{1}=E_{2}$, and write $\lambda$ for $\lambda_{1}=\lambda_{2}$. We conclude that $\mathrm{H}_{\lambda}\left(M_{1}\right) \cong \mathrm{H}_{\lambda}\left(M_{2}\right)$ as $\lambda$-adic Galois representations.

Write $\Lambda$ for the set of finite places of $E$. We assumed that $\mathrm{G}_{\ell}\left(M_{1} \oplus M_{2}\right)$ is connected for all prime numbers $\ell$. Because $\mathrm{H}_{\lambda}\left(M_{1}\right)$ and $\mathrm{H}_{\lambda}\left(M_{2}\right)$ are semisimple and quasi-compatible, Proposition 5.14 shows that $\mathrm{H}_{\Lambda}\left(M_{1}\right)$ and $\mathrm{H}_{\Lambda}\left(M_{2}\right)$ are isomorphic quasi-compatible systems of representations. We have now completed the first two steps of the strategy outlined in Section 9.2.

9.4. For $i=1,2$, let $\left(\Delta_{i}, \mu_{i}\right)$ be the Deligne-Dynkin diagram associated with $M_{i}$ as in Section 6.5. Let $f: \pi_{0}\left(\Delta_{1}\right) \rightarrow \pi_{0}\left(\Delta_{2}\right)$ be the map that is defined by the canonical identifications 


\section{MTC FOR PRODUCTS OF ABELIAN VARIETIES}

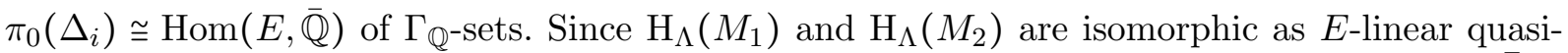
compatible systems of representations, we get local isomorphisms $\psi_{\ell} \in \operatorname{Isom}_{f}\left(\Delta_{1, \mathbb{Q}_{\ell}}, \Delta_{2, \mathbb{Q}_{\ell}}\right)^{\Gamma_{\mathbb{Q}_{\ell}}}$. By Lemma 7.4, we have $\psi_{\ell}\left(\mu_{1}\right)=\mu_{2}$ for all $\ell$. Thus we may apply Proposition 6.23 to obtain an isomorphism $\phi \in \operatorname{Isom}_{f}\left(\left(\Delta_{1}, \mu_{1}\right),\left(\Delta_{2}, \mu_{2}\right)\right)^{\Gamma_{\mathbb{Q}}}$ such that $\phi_{\lambda}=\psi_{\lambda}$ for some finite place $\lambda$ of $E$.

9.5. Let $S_{i}$ be the subset of $\mu_{i}$-symplectic nodes of $\left(\Delta_{i}, \mu_{i}\right)$. We will now apply Deligne's construction (see Section 8) to the motives $M_{1}$ and $M_{2}$. To do so, we have to make three choices, as in Section 8.2: (1) choose a totally imaginary quadratic extension $F / E$; then $(2)$ endow it with a partial CM type relative to $\left(\Delta_{1}, \mu_{1}\right) \stackrel{\phi}{=}\left(\Delta_{2}, \mu_{2}\right)$; finally, (3) choose the representations $V_{1}$ and $V_{2}$ in such a way that they have the same dimension.

Let $F_{S_{i}}$ be an étale $E$-algebra such that $\operatorname{Hom}\left(F_{S_{i}}, \overline{\mathbb{Q}}\right) \cong S_{i}$, and write $W_{F, i}$ for $F \otimes_{E} F_{S_{i}}$. Recall from Section 8.5 that $W_{F, i}$ carries a fractional Hodge structure of weight 1 that is of CM type. The construction produces two complex abelian varieties $A_{1}$ and $A_{2}$ such that $\mathrm{H}_{\mathrm{B}}\left(A_{i}\right)=W_{F, i} \otimes_{F_{S_{i}}} V_{i}$ and $M_{i, \mathbb{C}}=\mathrm{H}^{1}\left(A_{i}\right)^{\text {ha }}$. Replace $K$ with a finitely generated extension of $K$ such that $A_{1}$ and $A_{2}$ are defined over $K$. We are now ready for the two final steps in Section 9.2.

9.6. Let $\lambda$ be a finite place of $E$ such that $\psi_{\lambda}=\phi_{\lambda}$, and let $\ell$ be the residue characteristic of $\lambda$. Observe that $E$ acts on $M_{i}, V_{i}, W_{F, i}$, and $A_{i}$. We have the following diagram:

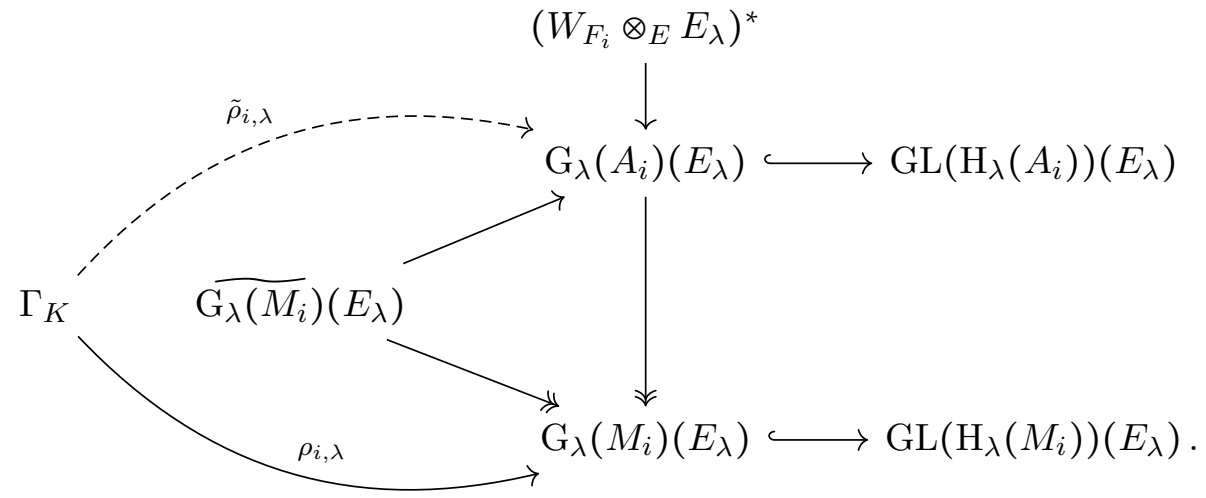

If we temporarily forget the dashed arrow $\tilde{\rho}_{i, \lambda}$, then we can identify the two diagrams for $i=1$ and $i=2$ to obtain a diagram

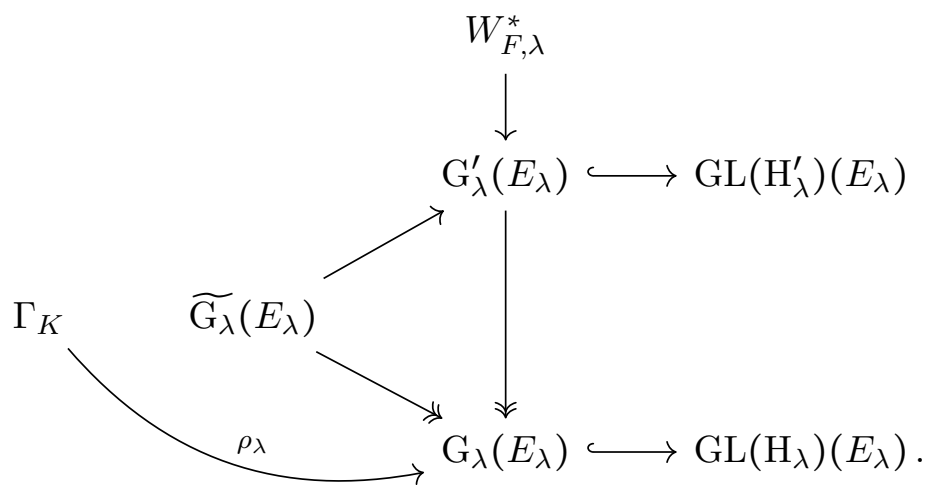

These identifications are justified as follows:

- In Section 9.3, we showed that there is an isomorphism $\mathrm{H}_{\lambda}\left(M_{1}\right) \rightarrow \mathrm{H}_{\lambda}\left(M_{2}\right)$ of $\lambda$-adic Ga- 


\section{J. Commelin}

lois representations identifying $\mathrm{G}_{\lambda}\left(M_{1}\right)$ with $\mathrm{G}_{\lambda}\left(M_{2}\right)$ and $\rho_{1, \lambda}$ with $\rho_{2, \lambda}$. We may also canonically identify $\overline{\mathrm{G}_{\lambda}\left(M_{1}\right)}$ and $\overline{\mathrm{G}_{\lambda}\left(M_{2}\right)}$. Call the resulting objects $\mathrm{H}_{\lambda}, \mathrm{G}_{\lambda}, \rho_{\lambda}$, and $\widetilde{\mathrm{G}_{\lambda}}$, respectively.

- This isomorphism also gives an isomorphism of Deligne-Dynkin diagrams $\psi_{\lambda}:\left(\Delta_{1}, \mu_{1}\right)_{\lambda} \rightarrow$ $\left(\Delta_{2}, \mu_{2}\right)_{\lambda}$ over $\mathbb{Q}_{\ell}$. In Section 9.4 , we showed that $\psi_{\lambda}$ extends to an isomorphism of DeligneDynkin diagrams $\phi:\left(\Delta_{1}, \mu_{1}\right) \rightarrow\left(\Delta_{2}, \mu_{2}\right)$ over $\mathbb{Q}$.

- This allows us to identify the $E$-algebras $W_{F, 1}$ and $W_{F, 2}$. Call the resulting algebra $W_{F}$ and write $W_{F, \lambda}$ for $W_{F} \otimes_{E} E_{\lambda}$.

- By Deligne's construction, we can identify $\mathrm{H}_{\lambda}\left(A_{1}\right)$ with $\mathrm{H}_{\lambda}\left(A_{2}\right)$ as representations of $\widetilde{\mathrm{G}_{\lambda}}$ with an action of $W_{F, \lambda}$. Call this representation $\mathrm{H}_{\lambda}^{\prime}$.

- Finally, $\mathrm{G}_{\lambda}\left(A_{i}\right)$ is the Zariski closure of the image of $W_{F, \lambda}^{*}$ and $\widetilde{\mathrm{G}_{\lambda}}$ in $\operatorname{GL}\left(\mathrm{H}_{\lambda}\left(A_{i}\right)\right)$. Thus, we may identify $\mathrm{G}_{\lambda}\left(A_{1}\right)$ with $\mathrm{G}_{\lambda}\left(A_{2}\right)$; call the resulting group $\mathrm{G}_{\lambda}^{\prime}$.

The dashed arrows $\tilde{\rho}_{1, \lambda}$ and $\tilde{\rho}_{2, \lambda}$ in the original diagrams can be identified with maps $\Gamma_{K} \rightarrow$ $\mathrm{G}_{\lambda}^{\prime}\left(\mathbb{Q}_{\ell}\right)$ that lift $\rho_{\lambda}$. It is our goal to show that they are identical.

9.7. To show that $\tilde{\rho}_{1, \lambda}$ and $\tilde{\rho}_{2, \lambda}$ are identical, we employ the following strategy: Recall that $\mathrm{G}_{\lambda}^{\prime}$ is a finite cover of $\left(\mathrm{G}_{\lambda}^{\prime}\right)^{\mathrm{ad}} \times\left(\mathrm{G}_{\lambda}^{\prime}\right)^{\mathrm{ab}}$. Since $\tilde{\rho}_{1, \lambda}$ and $\tilde{\rho}_{2, \lambda}$ lift the map $\rho_{\lambda}$, we know that their composition with the canonical quotient map $\mathrm{G}_{\lambda}^{\prime} \rightarrow\left(\mathrm{G}_{\lambda}^{\prime}\right)^{\text {ad }}$ is independent of $i=1$, 2 . If we also show that their composition with the canonical quotient map $G_{\lambda}^{\prime} \rightarrow\left(G_{\lambda}^{\prime}\right)^{\text {ab }}$ is independent of $i=1,2$, then we can show that $\tilde{\rho}_{1, \lambda}$ and $\tilde{\rho}_{2, \lambda}$ are identical, at least after replacing $K$ with a finite field extension of $K$. In executing this strategy, we carry out a minor variation.

9.8. Recall from Section 8.6 that for $i=1,2$, the natural map $p_{i}: \mathrm{G}_{\mathrm{B}}\left(W_{F}\right) \rightarrow \mathrm{G}_{\mathrm{B}}\left(A_{i}\right)^{\mathrm{ab}}$ is an isogeny. Therefore, there exists a torus $T$ together with isogenies $q_{i}: \mathrm{G}_{\mathrm{B}}\left(A_{i}\right)^{\mathrm{ab}} \rightarrow T$ such that $q_{1} \circ p_{1}=q_{2} \circ p_{2}$. Let $N$ be a faithful representation of $T$. Since $N$ is a representation of $\mathrm{G}_{\mathrm{B}}\left(W_{F}\right)$, we may canonically view $N$ as a fractional pre-Hodge structure, via the Tannakian formalism. However, since $N$ is a representation of $\mathrm{G}_{\mathrm{B}}\left(A_{i}\right)$, we know that $N$ is a (classical) Hodge structure, and this Hodge structure is independent of $i$ because $q_{1} \circ p_{1}=q_{2} \circ p_{2}$. Note that $N$ is a CM Hodge structure, by definition. Finally, by Theorem 4.13, we view $N$ as a complex CM motive.

Replace $K$ with a finitely generated extension of $K$ such that the motive $N$ is defined over $K$. Observe that the composite map

$$
\Gamma_{K} \stackrel{\tilde{\rho}_{i, \ell}}{\longrightarrow} \underbrace{\mathrm{G}_{\ell}\left(A_{i}\right)\left(\mathbb{Q}_{\ell}\right)}_{\mathrm{G}_{\mathrm{B}}\left(A_{i}\right)\left(\mathbb{Q}_{\ell}\right)} \longrightarrow \underbrace{\mathrm{G}_{\ell}\left(A_{i}\right)^{\mathrm{ab}}\left(\mathbb{Q}_{\ell}\right)}_{\mathrm{G}_{\mathrm{B}}\left(A_{i}\right)^{\mathrm{ab}}\left(\mathbb{Q}_{\ell}\right)} \stackrel{q_{i, \ell}}{\longrightarrow} \underbrace{\mathrm{G}_{\ell}(N)\left(\mathbb{Q}_{\ell}\right)}_{T\left(\mathbb{Q}_{\ell}\right)}
$$

defines the Galois representation on $\mathrm{H}_{\ell}(N)$ and therefore is independent of $i=1,2$.

Recall that we have quotient maps $\mathrm{G}_{\ell}\left(A_{i}\right) \rightarrow \operatorname{Res}_{E_{\lambda} / \mathbb{Q}_{\ell}} \mathrm{G}_{\lambda}\left(A_{i}\right)=\operatorname{Res}_{E_{\lambda} / \mathbb{Q}_{\ell}} \mathrm{G}_{\lambda}^{\prime}$, and therefore $\mathrm{G}_{\ell}\left(A_{i}\right)^{\mathrm{ab}} \rightarrow\left(\operatorname{Res}_{E_{\lambda} / \mathbb{Q}_{\ell}} \mathrm{G}_{\lambda}^{\prime}\right)^{\mathrm{ab}}$. We claim that there exists a finite quotient $\left(\operatorname{Res}_{E_{\lambda} / \mathbb{Q}_{\ell}} \mathrm{G}_{\lambda}^{\prime}\right)^{\mathrm{ab}} \rightarrow T^{\prime}$ such that the composite map

$$
\Gamma_{K} \stackrel{\tilde{\rho}_{i, \ell}}{\longrightarrow}\left(\operatorname{Res}_{E / \lambda / \mathbb{Q}_{\ell}} \mathrm{G}_{\lambda}^{\prime}\right)\left(\mathbb{Q}_{\ell}\right) \longrightarrow\left(\operatorname{Res}_{E_{\lambda} / \mathbb{Q}_{\ell}} \mathrm{G}_{\lambda}^{\prime}\right)^{\mathrm{ab}}\left(\mathbb{Q}_{\ell}\right) \longrightarrow T^{\prime}\left(\mathbb{Q}_{\ell}\right)
$$

is independent of $i=1,2$. Indeed, one may construct $T^{\prime}$ as follows: Let $K_{i}$ be the kernel of the isogeny $q_{i, \ell}: \mathrm{G}_{\ell}\left(A_{i}\right)^{\mathrm{ab}} \rightarrow \mathrm{G}_{\ell}(N)$, and let $K_{i}^{\prime}$ denote its image under the quotient map $\mathrm{G}_{\ell}\left(A_{i}\right)^{\mathrm{ab}} \rightarrow$ $\left(\operatorname{Res}_{E_{\lambda} / \mathbb{Q}_{\ell}} \mathrm{G}_{\lambda}^{\prime}\right)^{\mathrm{ab}}$. Then $K_{1}^{\prime} \cdot K_{2}^{\prime}$ is a finite subgroup of $\left(\operatorname{Res}_{E_{\lambda} / \mathbb{Q}_{\ell}} \mathrm{G}_{\lambda}^{\prime}\right)^{\mathrm{ab}}$, and we define $T^{\prime}$ as the quotient. By construction, there is a map $\mathrm{G}_{\ell}(N)\left(\mathbb{Q}_{\ell}\right) \rightarrow T^{\prime}\left(\mathbb{Q}_{\ell}\right)$, and the claim follows. 
9.9. We are almost done. Write $\pi^{\mathrm{ab}}$ for the map $\mathrm{G}_{\lambda}^{\prime}\left(E_{\lambda}\right)=\left(\operatorname{Res}_{E_{\lambda} / \mathbb{Q}_{\ell}} \mathrm{G}_{\lambda}^{\prime}\right)\left(\mathbb{Q}_{\ell}\right) \rightarrow T^{\prime}\left(\mathbb{Q}_{\ell}\right)$ and $\pi^{\mathrm{ad}}$ for the map $\mathrm{G}_{\lambda}^{\prime}\left(E_{\lambda}\right) \rightarrow\left(\mathrm{G}_{\lambda}^{\prime}\right)^{\mathrm{ad}}\left(E_{\lambda}\right)=\mathrm{G}_{\lambda}\left(E_{\lambda}\right)$. We have now proven that the compositions $\pi^{\mathrm{ab}} \circ \tilde{\rho}_{i, \lambda}$ and $\pi^{\mathrm{ad}} \circ \tilde{\rho}_{i, \lambda}$ do not depend on $i=1,2$.

Recall that $T^{\prime}$ is a quotient of $\left(\operatorname{Res}_{E_{\lambda} / \mathbb{Q}_{\ell}} G^{\prime}\right)^{\text {ab }}$ by a finite group. Hence, the group $\alpha=$ $\operatorname{ker}\left(\pi^{\mathrm{ad}}\right) \cap \operatorname{ker}\left(\pi^{\mathrm{ab}}\right)$ is a finite abelian subgroup of the centre of $\mathrm{G}_{\lambda}^{\prime}\left(E_{\lambda}\right)$. Define $\xi: \Gamma_{K} \rightarrow \mathrm{G}_{\lambda}^{\prime}\left(E_{\lambda}\right)$ via $\xi(g)=\tilde{\rho}_{1, \lambda}(g) \cdot \tilde{\rho}_{2, \lambda}(g)^{-1}$. This map $\xi$ takes values in $\alpha$ and is a homomorphism since $\alpha$ is contained in the centre of $\mathrm{G}_{\lambda}^{\prime}\left(E_{\lambda}\right)$. We conclude that after we replace $K$ with a finite extension, the homomorphisms $\rho_{1, \lambda}$ and $\rho_{2, \lambda}$ are the same. This means that $\mathrm{H}_{\lambda}\left(A_{1}\right)$ and $\mathrm{H}_{\lambda}\left(A_{2}\right)$ are isomorphic as $\lambda$-adic Galois representations. Another application of Proposition 5.14 shows that $\mathrm{H}_{\Lambda}\left(A_{1}\right)$ and $\mathrm{H}_{\Lambda}\left(A_{2}\right)$ are isomorphic $E$-rational quasi-compatible systems of representations. In particular, $\mathrm{H}_{\ell}\left(A_{1}\right)$ and $\mathrm{H}_{\ell}\left(A_{2}\right)$ are isomorphic Galois representations. Finally, Faltings's theorem ([Fal83, §5, Korollar 2], see also [Fal84]) implies that $A_{1}$ and $A_{2}$ are isogenous abelian varieties. Since $M_{i}=\mathrm{H}\left(A_{i}\right)^{\text {ha }}$, we conclude that $\mathrm{H}_{\mathrm{B}}\left(M_{1}\right)$ and $\mathrm{H}_{\mathrm{B}}\left(M_{2}\right)$ are isomorphic Hodge structures. This completes the proof of Proposition 9.1.

\section{The main theorem}

Lemma 10.1. Let $K$ be a field of characteristic 0 . For $i=1, \ldots, n$, let $G_{i}$ be a simple linear algebraic group over $K$. Let $G$ be a subgroup of $G_{1} \times \cdots \times G_{n}$ such that $G$ surjects onto $G_{i}$ for $1 \leq i \leq n$ and $G$ surjects onto $G_{i} \times G_{j}$ for $1 \leq i<j \leq n$. Then $G=G_{1} \times \cdots \times G_{n}$.

Proof. It suffices to prove the analogous statement for Lie algebras. This is precisely the lemma in Step 3 of [Rib76, Proof of Theorem 4.4.10, pp. 790-791].

Lemma 10.2. Let $K \subset \mathbb{C}$ be a finitely generated field. Let $M_{i}$, with $i \in I$, be a finite collection of irreducible hyperadjoint abelian motives over $K$. Write $M=\oplus M_{i}$. If $\operatorname{MTC}\left(M_{i}\right)$ is true for all $i \in I$, then $\operatorname{MTC}(M)$ is true.

Proof. By replacing $K$ with a finite extension, we may and do assume that $\mathrm{G}_{\ell}(M)$ is connected for all prime numbers $\ell$ and, in addition, that $M_{i}$ is geometrically irreducible for all $i \in I$. We also may and do assume that for $i, j \in I$, we have $M_{i} \cong M_{j}$ if and only if $i=j$. Recall that there is a natural injection $\mathrm{G}_{\ell}(M) \hookrightarrow \prod_{i \in I} \mathrm{G}_{\ell}\left(M_{i}\right)$, and the image projects surjectively onto the factors $\mathrm{G}_{\ell}\left(M_{i}\right)$. Since $\mathrm{G}_{\ell}(M)$ is connected, so is $\mathrm{G}_{\ell}\left(M_{i} \oplus M_{j}\right)$ for all $i, j \in I$. By Proposition 9.1, we know that if $i, j \in I$ are two different indices, then $\mathrm{G}_{\ell}\left(M_{i} \oplus M_{j}\right) \cong \mathrm{G}_{\ell}\left(M_{i}\right) \times \mathrm{G}_{\ell}\left(M_{j}\right)$; in other words, $\mathrm{G}_{\ell}(M)$ surjects onto $\mathrm{G}_{\ell}\left(M_{i}\right) \times \mathrm{G}_{\ell}\left(M_{j}\right)$. By Lemma 10.1, we conclude that $\mathrm{G}_{\ell}(M) \cong \prod_{i \in I} \mathrm{G}_{\ell}\left(M_{i}\right)$.

Theorem 10.3. Fix a finitely generated field $K \subset \mathbb{C}$. Let $\mathcal{M}_{K} \subset \operatorname{Mot}_{K}$ be the full subcategory of abelian motives for which the Mumford-Tate conjecture is true. Then the category $\mathcal{M}_{K}$ is a Tannakian subcategory of $\operatorname{Mot}_{K}$.

Proof. The category $\operatorname{Mot}_{K}$ is semisimple, so subquotients are direct summands. It is clear that the subcategory $\mathcal{M}_{K}$ is closed under duals, tensor powers, and direct summands. Let $M_{1}$ and $M_{2}$ be two objects in $\mathcal{M}_{K}$. We need to show that $M_{1} \oplus M_{2}$ and $M_{1} \otimes M_{2}$ are objects in $\mathcal{M}_{K}$. Observe that $M_{1} \otimes M_{2}$ is a direct summand of $\left(M_{1} \oplus M_{2}\right)^{\otimes 2}$. Thus we are done if we show that the Mumford-Tate conjecture is true for $M=M_{1} \oplus M_{2}$. By Proposition 4.19, it suffices to prove $\operatorname{MTC}\left(M^{\text {ha }}\right)$. Decompose $M^{\text {ha }}=\bigoplus_{i \in I} M_{i}^{\prime}$ into a sum of irreducible motives. Note that for every $i \in I$, the motive $M_{i}^{\prime}$ is hyperadjoint, and $\operatorname{MTC}\left(M_{i}^{\prime}\right)$ holds since the irreducible motive $M_{i}^{\prime}$ is a summand of $M_{1}^{\text {ha }}$ or $M_{2}^{\text {ha }}$ by Lemma 2.11(vi). Now the result follows from Lemma 10.2. 


\section{J. Commelin}

Remark 10.4. We give a short and incomplete list of examples of varieties for which it is known that their motives are objects of the category $\mathcal{M}_{K}$ of Theorem 10.3 (see [Moo17a] for a recent overview of the state of the art):

(i) geometrically simple abelian varieties of prime dimension [Tan84]

(ii) abelian varieties of dimension $g$ with trivial endomorphism ring over $\bar{K}$ such that $2 g$ is neither a $k$ th power for some odd $k>1$ nor of the form $\left(\begin{array}{c}2 k \\ k\end{array}\right)$ for some odd $k>1$ (see [Pin98, Theorem 5.14])

(iii) K3 surfaces [Tan91, Tan95]

(iv) cubic fourfolds [And96a, Theorem 1.6.1]

We remind the reader that the Mumford-Tate conjecture for an abelian fourfold $A$ with trivial endomorphism ring is still an open problem.

TheOrem 10.5. Let $K$ be a finitely generated subfield of $\mathbb{C}$. Let $A_{i}$, for $i \in I$, be a finite collection of abelian varieties over $K$, and write $A=\prod_{i \in I} A_{i}$. Assume that $\operatorname{MTC}\left(A_{i}\right)$ is true for all $i \in I$. Then $\operatorname{MTC}(A)$ is also true.

Proof. This is an immediate consequence of Theorem 10.3.

\section{ACKNOWLEDGEMENTS}

My warmest thanks go to my supervisor Ben Moonen. Our countless discussions and his many detailed explanations and corrections have been of immense importance for this article. I also thank Rutger Noot for a very inspiring discussion of this subject. This article also benefited from the extensive feedback on my PhD Thesis that I received from Anna Cadoret, Pierre Deligne, Bas Edixhoven, Milan Lopuhaä, Rutger Noot, and Lenny Taelman. I thank Netan Dogra, Brad Drew, Carel Faber, Salvatore Floccari, Martin Gallauer, Joost Nuiten, and Frans Oort for their interest and useful comments. I express my gratitude to an anonymous referee for detailed comments and feedback, and for catching an error in an earlier version of Section 6.9.

\section{REFERENCES}

And92 Y. André, Une remarque à propos des cycles de Hodge de type CM, Séminaire de Théorie des Nombres, Paris, 1989-90, Progr. Math., vol. 102 (Birkhäuser Boston, Boston, MA, 1992), 1-7; doi:10.1007/978-1-4757-4269-5_1.

And96a_ On the Shafarevich and Tate conjectures for hyper-Kähler varieties, Math. Ann. 305 (1996), no. 2, 205-248; doi:10.1007/BF01444219.

And96b_ Pour une théorie inconditionnelle des motifs, Publ. Math. Inst. Hautes Études Sci. 83 (1996), no. 1, 5-49; doi:10.1007/BF02698643.

Bre94 L. Breen, Tannakian categories, Motives (Seattle, WA, 1991), Proc. Sympos. Pure Math., vol. 55 (Amer. Math. Soc., Providence, RI, 1994), 337-376.

Com17 J. Commelin, On compatibility of the $\ell$-adic realisations of an abelian motive, Ann. Inst. Fourier (Grenoble), to appear, arXiv:1706.09444.

Del79 P. Deligne, Variétés de Shimura: interprétation modulaire, et techniques de construction de modèles canoniques, Automorphic Forms, Representations and L-Functions, Part 2 (Oregon State Univ., Corvallis, Ore., 1977), Proc. Sympos. Pure Math., vol. 33 (Amer. Math. Soc., Providence, R.I., 1979), 247-289. 


\section{MTC FOR PRODUCTS OF ABELIAN VARIETIES}

Del90 P. Deligne, Catégories tannakiennes, The Grothendieck Festschrift, Vol. II, Progr. Math., vol. 87 (Birkhäuser Boston, Boston, MA, 1990), 111-195; doi:10.1007/978-0-8176-4575-5_ 3.

DMOS82 P. Deligne, J. S. Milne, A. Ogus, and K. Y. Shih, Hodge cycles, motives, and Shimura varieties, Lecture Notes in Math., vol. 900 (Springer-Verlag, Berlin - New York, 1982); doi:10.1007/ 978-3-540-38955-2.

Fal83 G. Faltings, Endlichkeitssätze für abelsche Varietäten über Zahlkörpern, Invent. Math. 73 (1983), no. 3, 349-366; doi:10.1007/BF01388432.

Fal84_ Complements to Mordell, Rational Points (Bonn, 1983/1984), Aspects Math., vol. E6 (Friedr. Vieweg, Braunschweig, 1984), 203-227.

Fal88 , p-adic Hodge theory, J. Amer. Math. Soc. 1 (1988), no. 1, 255-299; doi:10.2307/ 1990970.

Kis17 M. Kisin, modp points on Shimura varieties of abelian type, J. Amer. Math. Soc. 30 (2017), no. 3, 819-914; doi:10.1090/jams/867.

Lom16 D. Lombardo, On the $\ell$-adic Galois representations attached to nonsimple abelian varieties, Ann. Inst. Fourier (Grenoble) 66 (2016), no. 3, 1217-1245; doi:10.5802/aif. 3035.

Mil13 J.S. Milne, Shimura varieties and moduli, Handbook of Moduli, Vol. II, Adv. Lect. Math., vol. 25 (Int. Press, Somerville, MA, 2013), 467-548.

Moo17a B. Moonen, Families of motives and the Mumford-Tate conjecture, Milan J. Math. 85 (2017), no. 2, 257-307; doi:10.1007/s00032-017-0273-x.

Moo17b On the Tate and Mumford-Tate conjectures in codimension 1 for varieties with $h^{2,0}=1$, Duke Math. J. 166 (2017), no. 4, 739-799; doi:10.1215/00127094-3774386.

Moo19_ A remark on the Tate conjecture, J. Algebraic Geom. 28 (2019), no. 3, 599-603; doi:10.1090/jag/720.

Pin98 R. Pink, l-adic algebraic monodromy groups, cocharacters, and the Mumford-Tate conjecture, J. reine angew. Math. 495 (1998), 187-237; doi:10.1515/crll.1998.018.

Poh68 H. Pohlmann, Algebraic cycles on abelian varieties of complex multiplication type, Ann. of Math. 88 (1968), no. 2, 161-180; doi:10.2307/1970570.

Rib76 K. A. Ribet, Galois action on division points of Abelian varieties with real multiplications, Amer. J. Math. 98 (1976), no. 3, 751-804; doi:10.2307/2373815.

Ser81 J.-P. Serre, Letter to K. Ribet (January 29, 1981), in Oeuvres - Collected Papers IV 1985-1998, Springer Collected Works in Math. (Springer-Verlag Berlin Heidelberg, 2000), 1-20.

Tan84 S. G. Tankeev, Cycles of abelian varieties of prime dimension over finite and number fields, Math. USSR Izv. 22 (1984), no. 2, 329-337; doi:10.1070/IM1984v022n02ABEH001446.

Tan91_ K3 surfaces over number fields and the Mumford-Tate conjecture, Math. USSR Izv. 37 (1991), no. 1, 191-208; doi:10.1070/IM1991v037n01ABEH002059.

Tan95_L Surfaces of type K3 over number fields and the Mumford-Tate conjecture. II, Izv. Math. 59 (1995), no. 3, 619-646; doi:10.1070/IM1995v059n03ABEH000026.

UY13 E. Ullmo and A. Yafaev, Mumford-Tate and generalised Shafarevich conjectures, Ann. Math. Qué. 37 (2013), no. 2, 255-284; doi:10.1007/s40316-013-0009-4.

Vas08 A. Vasiu, Some cases of the Mumford-Tate conjecture and Shimura varieties, Indiana Univ. Math. J. 57 (2008), no. 1, 1-75; doi:10.1512/iumj.2008.57.3513.

Johan Commelin jmc@math.uni-freiburg.de

Albert-Ludwigs-Universität Freiburg, Mathematisches Institut, Ernst-Zermelo-Straße 1, 79104 Freiburg im Breisgau, Germany 